\title{
Osthole Induces Apoptosis, Secondary Necrosis and Mitophagy Via NQ01-Mediated ROS Overproduction in HeLa Cells
}

Mengjie Huangfu

Guilin Medical University

Juan Wang

Guilin Medical University

Dan Yu

Guilin Medical University

Jianli Qin

Guilin Medical University

Xiao Guan

Guilin Medical University

Luwei Zhou

Guilin Medical University

Tong Dou

Guilin Medical University

Yisa Liu

Guilin Medical University

Lin Wang

Guilin Medical University

Xumei Li

Guilin Medical University

Minglei Fu

The Second Affiliated Hospital of Guilin medical university

xu chen ( $\nabla$ chenxu@glmc.edu.cn )

Guilin Medical University

\section{Research}

Keywords: Osthole, Cervical cancer, NQ01, ROS, Apoptosis, Secondary necrosis, Mitophagy

Posted Date: September 23rd, 2020

DOI: https://doi.org/10.21203/rs.3.rs-79478/v1 
License: (c) (i) This work is licensed under a Creative Commons Attribution 4.0 International License. Read Full License 


\section{Abstract}

Background: Osthole is a natural coumarin which has been proved to inhibit growth of cancer cells by inducing cancer cells death, while its mechanism of anticancer remains unclearly. In our study, we found that osthole activated multiple forms of cell death including apoptosis, secondary necrosis and mitophagy in receptor interacting protein kinase (RIP) 3-deficient cervical cancer HeLa cells.

Methods: Cell viability was detected by MTT assay. Cell membrane integrity was detected by LDH release assay and PI staining. Cell apoptosis and necrosis were detected by flow cytometry assay. Reactive oxygen species (ROS) was detected by DCFH-DA staining and mitochondrial membrane potential (MMP) was detected by JC-1 staining using flow cytometry. The expression of proteins was detected by western blotting assay and proteomics. Xenograft tumor model was used to evaluate the effect of osthole in vivo.

Results: Our study showed osthole caused HeLa cells apoptosis and secondary necrosis, which is a phenomenon of the apoptotic cells' plasma membrane breakdown. And when Hela cells pretreatment with Z-DEVD-FMK, an irreversible caspase-3 inhibitor, not only inhibited osthole-induced apoptosis but also necrosis. Moreover, we found that Z-DEVD-FMK reversed the effect of osthole on the induction of cleaved the N-terminal fragment of GSDME in Hela cells. Furthermore, inhibition of NAD (P) H: quinone oxidoreductase 1 (NQO1) by osthole induced the overproduction of reactive oxygen species (ROS). ROS inhibitor N-Acetyl-L-cysteine (NAC) not only reduced osthole-induced apoptosis, but also reversed its effect on the necrotic induction and the GSDME N-terminal generation. It was shown that osthole decreased mitochondrial membrane potential (MMP) and increased the expression of PTEN-induced putative kinase 1 (PINK1) and Parkin, which indicated that the activation of mitophagy induced by osthole. Meanwhile, as well as apoptosis and secondary necrosis, mitophagy was also restrained by NAC.

Conclusions: In conclusion, all these data suggested that osthole induced apoptosis, secondary necrosis and mitophagy via NQ01-mediated ROS overproduction.

\section{Background}

Cervical cancer (CC) is the fourth most common cancer in women worldwide and the mortality rate of CC is also ranked fourth in female tumors[1]. Chemotherapy is the standard treatment for advanced or recurrent patients. However, the occurrence and development of chemotherapy resistance may affect the treatment effect[2]. Therefore, finding effective drugs is urgent for cervical cancer therapy.

Osthole, a natural coumarin extracted from the Cnidium monnieri (L.) Cusson. (Fig. 1A), has been shown to have inhibitory effect in multiple sorts of cancers, including breast cancer[3], ovarian cancer[4] and hepatic carcinomas[5]. In addition, osthole is used as an adjunct therapy to conquer cisplatin resistance and enhance radiotherapy sensitivity in cervical cancer cells[6, 7], and it induced apoptosis in cervical cancer cells[8]. However, there are few studies to evaluate its cell death induction of other forms. Thus, 
our study attempts to reveal whether osthole induces cell death besides apoptosis and explore its potential mechanism.

Secondary necrosis is a phenomenon of apoptotic cells' plasma membrane integrity lost, and one way is triggered by GSDME (DFNA5). GSDME is a member of gasdermin superfamily and could switch caspase3-mediated apoptosis to secondary necrosis[9]. The N-terminal domain of GSDME (GSDME-N) is cleaved by apoptotic activated caspase-3, which causes the induction of secondary necrosis[9]. Mitophagy is a selective form of autophagy, which degraded damaged mitochondria in autophagolysosome to maintain intracellular homeostasis[10]. The PINK1/Parkin pathway is the critical pathway mediate mitophagy[11]. Different PCDs have different cell morphologies and molecular mechanisms, and some of them were one consequence of ROS overproduction in cells[12]. The level of ROS in cancer cells is higher than normal cells, but when the production of ROS rises to the level of cytotoxicity, the cancer cells can be suppressed, even induced to death[13]. Though ROS provides a common pathway leading to mitophagy, apoptosis and necrosis, the interrelationships among them remains an unclearly, and how these three pathways are regulated by osthole remain unknown.

\section{Material And Methods}

\section{Reagents and antibodies}

Osthole and cisplatin were purchased from Meilunbio (purity $\geq 98 \%$, Dalian, China). Dicoumarol (DIC), ZVAD-FMK, Z-DEVD-FMK and 3-Methyladenine (3-MA) were purchased from MedChem Express (Princeton, NJ, USA). N-Acetyl-L-cysteine (NAC) and JC-1 were purchased from Beyotime (Shanghai, China). Dimethyl sulfoxide (DMSO) and 2', 7'-Dichlor-ofluorescin diacetate (DCFH-DA) were purchased from Sigma-Aldrich (St. Louis, MO, USA). Anti-rabbit $\lg G$, anti-mouse $\lg G$ and antibodies against $\beta$-actin were purchased from ZSGB-BIO (Beijing, China). Anti-LC3A/B antibody was purchased from Cell Signaling Technology (Danvers, MA, USA). Anti-NQ01, anti-caspase-3, anti-cleaved caspase-3, anti-PARP-1 and anti-GSDME antibodies were purchased from Abcam (Cambridge, MA, USA). FITC Annexin V Apoptosis Detection Kit (BD Pharmingen, CA, USA)

\section{Cell cultures}

The human cervical cancer HeLa cell line and normal liver cell line LO2 were obtained from Conservation Genetics CAS Kunming Cell Bank (Kunming, China). The cells were cultured in DMEM (Gibco, Grand Island, NY, USA) supplemented with 10\% fetal bovine serum (FBS; Gibco, Auckland, New Zealand), 100 $\mathrm{U} / \mathrm{mL}$ penicillin and $100 \mu \mathrm{g} / \mathrm{mL}$ streptomycin (Solarbio, Beijing, China), and maintained in a humid environment with $5 \% \mathrm{CO}_{2}$ at $37^{\circ} \mathrm{C}$.

\section{Cell viability assay}

Hela cells were seeded into 96-well plates at 4000 cells/well and incubated overnight. After that, cells were treated with osthole at indicated concentrations for indicated times. Then, $20 \mu \mathrm{L}$ MTT (Solarbio, 
Beijing, China) was added to each well and the plate was incubated for $4 \mathrm{~h}$ at $37^{\circ} \mathrm{C}$. The formed formazan crystals were dissolved with $150 \mu \mathrm{L}$ DMSO, and the absorbance was detected at $490 \mathrm{~nm}$ with a microplate reader(TECANखSwitzerland).

\section{Lactate dehydrogenase (LDH) release assay}

The LDH release was detected by LDH assay kit (Nanjing Jiancheng Bioengineering Institute, Nanjing, China). According to the instruction, cell culture supernatant was collected and added the corresponding reagents. The absorbance was measured at $450 \mathrm{~nm}$ with a microplate reader.

\section{Flow cytometry analysis of Annexin V-FITC/PI staining}

Osthole treated cells were harvested and washed by cold phosphate-buffered saline (PBS) thrice. The cells were suspended in $200 \mu \mathrm{L} 1 \times$ binding buffer containing $5 \mu \mathrm{L}$ Annexin V-FITC and $5 \mu \mathrm{L} \mathrm{PI}$ and incubated for $30 \mathrm{~min}$ in the dark. After that, $100 \mu \mathrm{L} 1 \times$ binding buffer was supplemented to each sample, and the cells were detected by flow cytometer (BD Biosciences, CA, USA).

\section{Measurement of ROS}

Intracellular ROS levels were determined using the fluorogenic probe DCFH-DA. After treatment with osthole, the cells were washed with PBS and dyed by DCFH-DA $(2 \mu \mathrm{M})$ for $30 \mathrm{~min}$ at $37^{\circ} \mathrm{C}$ in the dark. The cells were harvested and the level of ROS was measured by flow cytometry (BD Biosciences, USA).

\section{Hoechst 33342 and PI staining}

HeLa cells were cultured in 6-well plates for $24 \mathrm{~h}$ and pretreated with osthole for $18 \mathrm{~h}$. Then, cells were washed with PBS and stained by Hoechst $33342(1 \mu \mathrm{g} / \mathrm{ml})$ for $5 \mathrm{~min}$, following incubation with PI (5 $\mu \mathrm{g} / \mathrm{ml}$ ) for $15 \mathrm{~min}$ at room temperature. The cells were observed under cell imaging station (ThermoFisher Scientific, Bonn, Germany) after washed with PBS.

\section{Measurement of MMP}

Mitochondrial membrane potential was determined by the fluorescent probe JC-1 (Beyotime Biotech, Nanjing, China). The cells were suspended in PBS containing $0.2 \mu \mathrm{M} \mathrm{JC}-1$. After incubated for 20 min in the dark, the samples were analyzed by flow cytometry. JC-1 existed as a polymer when the MMP is high otherwise exists as a monomer, and the two groups of cells can be distinguished by flow cytometry (BD Biosciences, USA).

\section{MDC staining assay}

MDC staining assay was used to detect autolysosome. After treatment with osthole, the cells were incubated with $50 \mu \mathrm{M} \mathrm{MDC}$ for 30 min at $37^{\circ} \mathrm{C}$. Then, the cells were washed three times with PBS and observed under fluorescence microscopy straightway (Olympus, Tokyo, Japan). 


\section{Label-Free Quantitative Proteomics}

Protein extraction and peptide separation were performed as previous described method[14]. MS experiments were performed on a Q Exactive mass spectrometer that was coupled to Easy nLC (ThermoFisher Scientific, Bonn, Germany). MS data was acquired using a data-dependent top 10 method dynamically choosing the most abundant precursor ions from the survey scan (350-1800 m/z) for HCD fragmentation. The instrument was run with peptide recognition mode enabled. MS experiments were performed triply for each sample.

\section{Bioinformatics analysis of differentially expressed proteins}

The MS raw data were processed using MaxQuant software version 1.5.5.1 and quantified by label free quantitation (LFQ) [15]. Gene Ontology (GO) enrichment analysis with biological process, molecular function and cellular components of potential targets were carried out for biological function annotation based on a bioinformatics database (http://geneontology.org/). KOALA (KEGG Orthology and Links Annotation) software was used to analyze KEGG Pathway database. Fisher's Exact Test was used to the distribution of GO classification or KEGG pathway in target protein collection and total protein collection.

\section{Western blotting analysis}

The cells were harvested and lysed with RIPA lysate buffer for 30 minutes on ice. After centrifuged, the supernatant was obtained and quantified using BCA kit. The protein was separated by SDS-PAGE electrophoresis and transferred to a NC membrane. These membranes were blocked with $5 \%$ skim milk for $2 \mathrm{~h}$ and were incubated with primary antibody at $4{ }^{\circ} \mathrm{C}$ overnight. After that, membranes were incubated with horseradish peroxidase-conjugated secondary antibody $(1: 4000)$ for $1 \mathrm{~h}$ at room temperature. Protein bands were detected by Western ECL substrates (Bio-Rad, CA, USA) and gray values were analyzed by Image $\mathrm{J}$.

\section{Xenograft assay}

The use of all mice in this study was approved by the Institutional Animal Care and Use Committee of Guilin Medical University. Female BALB/c-nude mice (4 weeks old) were purchased from Hunan SJA Laboratory Animal Co., Ltd. Hela cells $\left(1^{\prime} 10^{7}\right)$ were injected subcutaneously into the right flanks of the mice. When the tumor volume reached $50 \mathrm{~mm}^{3}$, nude mice were intraperitoneally injected with osthole (150 mg/kg, dissolved in corn oil), and control group mice were injected with corn oil (Sigma, St. Louis, MO, USA). The length and width of the tumor was measured every two days using vernier calipers (Volume= length ' width $\left.{ }^{2} / 2\right)$. Following treatment for 21 days, the mice were sacrificed, and the weights of xenograft tumors were measured. After that, the total protein from the excised tumor tissues was extracted by RIPA lysis buffer. The expression of proteins was detected by western blotting analysis.

\section{Statistical analysis}


All data represent at least three independent experiments and was analyzed by GraphPad Prism 8 software (GraphPad, San Diego, CA). The results were expressed as mean \pm standard deviation (SD). Differences among groups were compared using a two-tailed Student's t-test or one-way ANOVA. $P<0.05$ was considered statistically significant.

\section{Results}

\section{Osthole inhibited cell viability and induced cell death in HeLa cells}

In order to detect the effect of osthole on cell viability, HeLa cells were treated with indicated concentrations of osthole for indicated time. Our result showed that osthole induced dose- and timedependent reduction of cell viability in HeLa cells (Fig 1A), and $400 \mu \mathrm{M}$ osthole did not shown obviously inhibition on normal LO2 cells (Fig 1C). The phase-contrast images showed that osthole-treated cells appeared morphological characteristics of dying cells. (Fig 1D). Thus, osthole not only inhibited the cell viability, but also induced HeLa cells death.

\section{Osthole induced apoptosis and necrosis in RIP3-deficient cells}

To figure out the death manner of HeLa cells caused by osthole, we detected the release of LDH which indicates a breakdown of the cell membrane. The result showed that the LDH release induced by osthole was in a dose-dependent manner (Fig 2A). Annexin FITC/PI staining showed that the apoptosis rate (Q2 and Q3 quadrant) was increased in a dose-dependent manner accompanied with necrosis rate increased (Fig 2B and C). Moreover, a typical apoptosis inducer cisplatin was used to induce apoptosis as a positive control. We found that cisplatin-induced cell death is concentrated in Q3 quadrant, while osthole treated cells exhibited an apparent increase in both the apoptotic and necrosis population (Fig 2D). To further determine whether osthole-induced cell necrosis is associated with necroptosis, we used necrostatin-1 (Nec-1), a RIP1 inhibitor which could specific inhibited necroptosis occurs. We found that pretreated with Nec-1 reversed osthole's inhibition on cell viability (Fig 2E). However, the expression of RIP3, a key necroptosis factor, couldn't be detected in HeLa cells (Fig 2F), which is consistent with the previously reported results[16]. These results indicated that osthole induced Hela cells death through apoptosis and RIP3-independent necrosis pathway, which raised our great interest.

\section{Osthole induced caspase-related apoptosis and necrosis in HeLa cells}

In order to confirm osthole induced apoptosis, we detected two indicators of apoptosis caspase-3 and PARP-1's expression. We found that osthole increased the expression of cleaved caspase-3 and cleaved PARP-1 in dose- and time-dependent manners (Fig 3A and B). Pretreatment with pan caspase inhibitor ZVAD-FMK prevented osthole-induced decrease in cell viability (Fig 3C). Further analysis of Annexin V-FITC and PI staining showed that Z-VAD-FMK reduced osthole-induced apoptosis (Q2 and Q3 quadrant). To our surprise, pretreatment with Z-VAD-FMK decreased the osthole-induce necrosis rate raise as well (Fig $3 \mathrm{D}$ and E). All above results suggested that osthole-induced RIP3-deficient Hela cells necrosis was related with caspase activation. 


\section{Osthole induced caspase-3 dependent secondary necrosis in HeLa cells}

As shown in Fig 1D, the phase-contrast images showed that the dying cells blew characteristic large bubbles from the plasma membrane which was the typical characteristics of pyroptosis or secondary necrosis. To further explore whether osthole-induced necrosis was induced by pyroptosis or secondary necrosis, Annexin $\mathbb{B}-\mathrm{FITC} / \mathrm{PI}$ and Hoechst 33342/PI dual staining were used to confirm the membrane

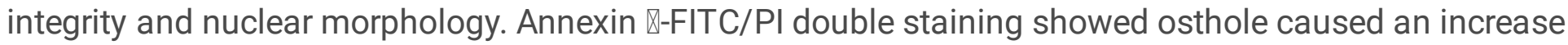
in $\mathrm{PI}^{+}$cells (Q1 and Q2 quadrant) but decrease in increased $\mathrm{PI}^{-}$cells (Fig 4A and B), while Hoechst 33342/ $\mathrm{PI}$ dual staining proved that PI positive cells treated by osthole have typical apoptosis features, such as nuclear condensation or fragmentation (Fig 4C). Thus, all these data suggested that osthole-induced necrosis was secondary to apoptosis.

Our western blotting confirmed that the expression of GSDME-N in osthole-treated cells were significantly higher than in untreated cells and raised greatly (Fig 4D). We also found that caspase-3 inhibitor Z-DEVD partially reversed the proliferation inhibition, apoptosis and necrosis induction caused by osthole (Fig 4EH). To further explore the implication of caspase- 3 in apoptosis and secondary necrosis, related proteins were analyzed by western blotting. It was shown that the increased levels of cleaved caspase-3 and cleaved PARP-1 expression were overturned, while the raised secondary necrosis biomarker GSDME-N was reserved in protein expression as well (Fig $4 \mathrm{I}$ and $\mathrm{J}$ ). In summary, these results suggested that osthole induced caspase-3-related apoptosis and secondary necrosis.

\section{Proteomic analysis of osthole treated HeLa Cells}

To identify the differentially expressed proteins which related with osthole's effect on apoptosis and secondary necrosis induction in HeLa cells, label-free quantitative proteomics technique was used. A total of 4297 proteins were identified (Table S1). We screened out a total of 3015 proteins of which 39 proteins in osthole-treated group and 45 proteins in control group with 2931 proteins in common (Fig 5A). The differential expression between the osthole-treated group and control group was presented by volcano plot (Fig 5B). According to $P \otimes 0.05$ and fold-change $\otimes 1.5$ or $\otimes 0.67$ as the threshold, 44 differentially expressed proteins were screened and 21 proteins were up- regulated and 23 proteins were downregulated under osthole treated(Table S2). An unsupervised hierarchical clustering of the differential expressed proteins based on LFQ intensities is represented as a heatmap (Figure 5C). As Figure 5D showed that the 10 most significant cellular component, molecular function and biological process were changed by osthole in Hela cells. Regarding cellular component, proteins were mainly involved in the mitochondrial-related respiratory chain complex IV, mitochondrial intermembrane space, and succinateCoA ligase complex and autophagy-related amphisome. Based on molecular function, proteins were chiefly attributed to mitochondrial-related cytochrome-c oxidase activity, argininosuccinate synthase activity and phosphoenolpyruvate carboxykinase (GTP) activity (Fig 5D). Moreover, KEGG database was used to enrich differentially expressed proteins, the top 10 pathways were screened (Figure 5E). Next, we found that the dysregulated protein succinate-CoA ligase GDP/ADP-forming subunit alpha (SUCLG1), phosphoenolpyruvate carboxykinase 2 (PCK2), argininosuccinate synthase 1 (ASS1), coiled-coil-helix- 
coiled-coil-helix domain containing 2 (CHCHD2), SURF1 cytochrome c oxidase assembly factor (SURF1), and NADH: ubiquinone oxidoreductase subunit A9 (NDUFA9) were related to function of mitochondria (Fig 5F-G). Thus, according to the above analysis, we speculated that osthole caused cell death may be closely related to the function change of mitochondria.

\section{Osthole induced caspase-3 mediated mitophagy via PINK1/Parkin pathway in HeLa cells}

In order to elucidate the effect of osthole on mitochondria function, we firstly detected the MMP by JC-1 staining using flow cytometry. The result showed that the red fluorescence of $\mathrm{JC}-1$ transform to green fluorescence as the osthole concentration increased (Fig 6A and B), and the autophagy inhibitor 3methyladenine (3-MA) was significantly reserved the loss of cell vitality in HeLa cells. Moreover, the expression of PINK, Parkin and LC3区/ 『were higher in osthole treated group than in control group (Fig 5D). To explore the effect of caspase-3 on osthole-induced mitophagy, we pretreated HeLa cells with Z-DEVD. According to MDC staining, after pretreatment with Z-DEVD, the number of autophagic vacuoles was reduced and the fluorescent of MDC was weakened. Furthermore, the up-regulated PINK1, Parkin and LC3囚/囚 were inhibited by Z-DEVD in Hela cells. Thus, these results indicated that osthole induced mitophagy was related with the activity of caspase-3 in HeLa cells.

\section{NQ01 contributed to the osthole-induced apoptosis, secondary necrosis, mitophagy and the generation of ROS}

Previous reports showed that osthole could reduce NQO1 expression in cervical cancer SiHa and CaSki cells [6]. Our proteomics analysis showed that osthole-treated HeLa cells expressed lower NQ01 than control group (Fig 7A). Pretreatment with NQ01 inhibitor dicoumarol (DIC) strengthened the cell viability inhibition of osthole (Fig 7B). It has been reported that inhibiting NQ01 could increase ROS production [17]. In this study, we found that osthole treatment also triggered excessive production of ROS in HeLa cells, and DIC pretreated further strengthened the generation of ROS (Fig 7C). Furthermore, we found that the expression of NQ01 was first reduced then increased to normal level in osthole treated cells (Fig 7D). And the level of cellular ROS in osthole-treated cells increased first and then returned to normal level (Fig 7E). These results demonstrated that inhibited NQO1 expression augmented ROS level, while elevated NQ01 expression attenuated ROS production. In addition, we used DIC to reduce NQ01 expression in osthole treated cells. We found that DIC pretreatment enhanced the expression of cleaved caspase- 3 and cleaved PARP-1, and GSDME-N (Fig 7F and G). Moreover, the expression of PINK1, Parkin and LC3区/ upregulated by osthole were also enhanced in pretreated DIC cells (Fig $7 \mathrm{H}$ and I). Thus, these results suggested that NQO1 inhibition contributed to osthole-induced cell death and ROS overproduction.

ROS played a vital role in osthole-induced apoptosis, secondary necrosis and mitophagy

Due to NQ01 inhibition could induce overproduction of ROS, we wonder whether ROS plays an important role in osthole-induced cell death. Firstly, we found that pretreated with NAC, a ROS inhibitor blocked the production of ROS caused by osthole in Hela cells (Fig 8A). Then, the JC-1 staining was used to detect the effect of ROS on MMP lost caused by osthole. We observed that pretreated with NAC weakened 
osthole-induced mitochondrial depolarization (Fig 8B). Moreover, pretreatment with NAC significantly inhibited osthole-induced decrease in cell viability (Fig $8 \mathrm{C}$ ). According to the result of Annexin $\mathrm{X}$-FITC/PI staining, we found that NAC nearly inhibited osthole-induced apoptosis and secondary necrosis (Fig 8D and E). Western blotting analysis revealed that NAC attenuated osthole caused upregulation of cleaved caspase-3, cleaved PARP-1 and GSDME-N (Fig 8F). Concurrently, the up-regulated proteins PINK1, Parkin and LC3区/ induced by osthole were declined by NAC (Fig 8G). Thus, all these results suggested that ROS played a significant role in osthole-induced cell death.

\section{Osthole suppressed HeLa xenograft tumor growth in vivo}

To explore the antitumor effect of osthole on cervical cancer in vivo, HeLa cells were xenografted into BALB/c nude mice. We found that nude mice body weight in osthole treated group had no significant changed compared with control group (Fig 9A). Meanwhile, HE staining showed that osthole-treated livers displayed no pathological changes compared with control group (Fig S1). Moreover, after treated with osthole 21 days, the tumor size and tumor weight of nude mice were all significantly reduced than the control group (Fig 9B, C and D), and $150 \mathrm{mg} / \mathrm{kg}$ osthole significantly decreased tumor weight nearly by $75 \%$.. Further, western blotting analysis revealed that osthole treatment increased the protein expression

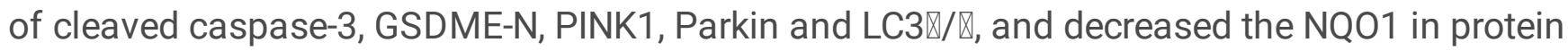
expression (Fig 9E and F). These results suggested that osthole $(150 \mathrm{mg} / \mathrm{kg})$ suppressed the tumor proliferation and exerted little effects on nude mice body weight and liver. The mechanism of osthole induced cell apoptosis $₫$ secondary necrosis and mitophagy may be associate with the regulation of NQ01 pathway.

\section{Discussion}

In this study, we clarified the underlying mechanism on osthole-induced cell death, including apoptosis, secondary necrosis and mitophagy in RIP3-deficient HeLa cells in vitro and in vivo. Our data suggested that osthole significantly increased the production of ROS by inhibiting the expression of NQO1 in HeLa cells. Moreover, the generation of ROS induced the mitochondrial depolarization and activated the caspase-3. Subsequently, activated caspase-3 induced apoptosis and further switched apoptosis to secondary necrosis. We also found that activated caspase-3 mediated mitophagy by PINK1/Parkin signal pathways. Thus, our findings illustrated that osthole induced multiple PCDs via ROS overproduction in HeLa cells.

Previous literature reported that osthole induced cell death mainly in the form of apoptosis[18-20]. A recent study demonstrated that osthole induced cell death through the GSDME-dependent pyroptosis pathway[21]. GSDME is a member of the gasdermins family which contains a cytotoxic $\mathrm{N}$ - terminal domain to form pore and induce membrane permeabilization. The activation of caspase- 3 mediates the occurrence of apoptosis, and specifically cleaves GSDME, which generates a GSDME-N fragment to induce secondary necrosis/pyroptosis [22]. It has been reported that GSDME-induced pyroptosis after apoptosis is considered as secondary necrosis[9]. In addition, caspase-3 inhibitor Z-DEVD reversed 
osthole induced apoptosis and necrosis which also indicated that osthole caused secondary necrosis via GSDME-N in HeLa cells. Our study is consistent with previous report that osthole-induced necrosis was secondary to apoptosis[9, 23].

NQ01 was high expressed in diverse human cancers, including breast cancer, colon cancer, cervical cancer and lung cancer[24]. It is reported that the role of NQO1 in cancer cells is to prevent excessive oxidative stress from damaging cells which is consistent with that in normal cells. So, NQ01 expression inhibited can disrupt intracellular redox balance in cancer cells [17, 25]. It has reported that osthole inhibited the increase of NQ01 expression to overcome cisplatin resistance in cervical cancer $\mathrm{SiHa}$ and CaSki cells [6]. And our proteomics analysis showed that many dysregulated proteins were closely related with mitochondria function included NQ01. Considering these reports and our proteomics profiling, we evaluated the relationship between NQ01 expression and the death induction of osthole on Hela cells as well as the production of ROS. Interestingly, we found that osthole suppressed the expression of NQ01 and NQ01 inhibitor DIC enhanced the apoptosis, secondary necrosis and mitophagy induction of osthole on Hela cells. Moreover, osthole decreased NQ01 expression which caused ROS generation elevated in Hela cells, and with the restoration of NQO1 expression, the level of ROS also gradually approached the normal level compared with untreated cells in HeLa cells.

It has been reported that osthole-induced antitumor effects is relevant to ROS overproduction[26]. Although different forms of PCDs have different molecular mechanisms, it seems that they cannot bypass the key link of ROS generation[12]. When the upstream caspase-3 activated, ROS generation plays an essential role in its mediation of apoptosis [27]. Overproduction of ROS also mediates DNA damage and activates PARP-1, which is downstream of activated caspase-3 [28]. Moreover, Yu et al. demonstrate that ROS plays a critical function in GSDME-mediated cell death[29]. Our study indicated that ostholeinduced ROS overproduction mediated the events of apoptosis and secondary necrosis. The excessive production of ROS is often accompanied with the loss of MMP $(\Delta \psi \mathrm{m})$ which implies mitochondrial damage and used as a hint for mitophagy[30]. Mitophagy, a selective form of autophagy, exists as an approach to clear damaged mitochondria and maintains mitochondrial homeostasis[31]. PINK1/Parkin is the most widely studied mitophagy pathway. As the initiator of mitophagy, PINK1 accumulates at the outer mitochondrial membrane to response mitochondrial depolarization and recruits Parkin to the outer mitochondrial membrane. Then, PINK1 and Parkin interact with processed LC3 to target mitochondria for degradation[11]. In this study, we found that osthole increased the expression of PINK1 and Parkin as well as the level of LC3区/ in vitro and in vivo, and ROS inhibitor NAC restrained osthole's effect on mitophagy induction. It has reported that mitophagy eliminated the damaged mitochondria to cut back the generation of ROS[32, 33]. Thus, we conjectured that the restoration of NQ01 expression and ROS production is related to the mitophagy induced by osthole. Further study will be focus on the interconnection between PINK1/Parkin pathway and NQ01 expression.

\section{Conclusions}


In summary, as depicted in Fig. 10, ROS mediated the occurrence of diverse cell deaths, including apoptosis, secondary necrosis and mitophagy in osthole-treated HeLa cells, and NQ01 expression play an important role in the death induction and ROS generation caused by osthole. However, further studies should be performed to explore more mechanisms about osthole's effect on death induction. It is anticipated that osthole may be a potential therapeutic agent for cervical cancer treatment.

\section{Abbreviations}

RIP3: Receptor interacting protein kinase; NQ01:NAD (P) H: quinone oxidoreductase 1;NAC: N-Acetyl-Lcysteine; ROS: Reactive oxygen species; MMP: Mitochondrial membrane potential; PINK1: PTEN-induced putative kinase 1 ; GSDME : Gasdermin E; CC: Cervical cancer; DCFH-DA: 2', 7'- Dichlor-ofluorescin diacetate; LDH: Lactate dehydrogenase; FBS: Fetal bovine serum; PI: Propidium lodide; 3-MA: 3Methyladenine; DMSO: Dimethyl sulfoxide; JC-1: 5,5',6,6'-tetrachloro-1,1',3,3'tetraethylbenzimidazolylcarbocyanine iodide.

\section{Declarations}

\section{Acknowledgements}

Not applicable.

\section{Authors' contributions}

HFMJ, WJ and CX participated in the design of the experiment. HFMJ, QJL, YD, GX, DT and LXM performed the experiments. WJ and WL wrote the paper and revised the manuscript. FML, ZLW and LYS were involved in data analysis and picture layout.

\section{Funding}

This work was supported by the National Natural Science Foundation of China (No. 81760443 and No. 81760663), Guangxi Special Fund Project for Innovation-Driven Development (GuikeAA19254025), the Project of Guangxi Natural Science Foundation (No. 2017GXNSFDA198 029), the Fourth Batch of Bagui Scholars' Special Funds for 2017(NO. [2017] 143), the Small Talent Highland Fund in Guangxi (No.201707), and the Scientific Research and Technology Development Program of Guilin (No.2017010938).

\section{Availability of data and materials}

Not applicable

\section{Ethics approval and consent to participate}


The use of all mice in this study was approved by the Institutional Animal Care and Use Committee of Guilin Medical University (Guilin, Guangxi, China).

\section{Consent for publication}

Not applicable.

\section{Competing interests}

The authors declare that they have no conflict of interests.

\section{Author details}

${ }^{a}$ College of Pharmacy, Guilin Medical University, 541004 Guilin, China; ${ }^{b}$ School of Basic Medical Sciences, Guilin Medical University, Guilin 541004, China; ${ }^{c}$ XiangYa School of Medicine of Central South University, 410013 Hunan, China; ${ }^{d}$ Dispensary, The Second Affiliated Hospital of Guilin Medical University.

\section{References}

1. Bray F, Ferlay J, Soerjomataram I, Siegel RL, Torre LA, Jemal A. Global cancer statistics 2018 : GLOBOCAN estimates of incidence and mortality worldwide for 36 cancers in 185 countries. CA Cancer J Clin. 2018;68:394-424.

2. Zhu H, Luo H, Zhang W, Shen Z, Hu X, Zhu X. Molecular mechanisms of cisplatin resistance in cervical cancer. Drug Des Devel Ther. 2016;10:1885-95.

3. Dai X, Yin C, Zhang Y, Guo G, Zhao C, Wang O, et al. Osthole inhibits triple negative breast cancer cells by suppressing STAT3. J Exp Clin Cancer Res Journal of Experimental Clinical Cancer Research. 2018;37:1-11.

4. Jiang G, Liu J, Ren B, Tang Y, Owusu L, Li M, et al. Anti-tumor effects of osthole on ovarian cancer cells in vitro. J Ethnopharmacol. 2016;193:368-76.

5. Lin ZK, Liu J, Jiang GQ, Tan G, Gong P, Luo HF, et al. Osthole inhibits the tumorigenesis of hepatocellular carcinoma cells. Oncol Rep. 2017;37:1611-8.

6. 10.1016/j.bbrc.2019.04.021

Su J, Zhang F, Li X, Liu Z. Osthole promotes the suppressive effects of cisplatin on NRF2 expression to prevent drug-resistant cervical cancer progression. Biochem Biophys Res Commun [Internet]. Elsevier Ltd; 2019;514:510-7. Available from: https://doi.org/10.1016/j.bbrc.2019.04.021.

7. Che Y, Li J, Li Z, Li J, Wang S, Yan Y, et al. Osthole enhances antitumor activity and irradiation sensitivity of cervical cancer cells by suppressing ATM/NF-?B signaling. Oncol Rep. 2018;40:737-47.

8. Chou S-Y, Hsu C-S, Wang K-T, Wang M-C, Wang C-C. Antitumor effects of Osthol fromCnidium monnieri: an in vitro andin vivo study. Phyther Res [Internet]. 2007;21:226-30. Available from: http://www3.interscience.wiley.com/journal/117934759/abstract. 
9. $10.1038 /$ ncomms 14128

Rogers C, Fernandes-Alnemri T, Mayes L, Alnemri D, Cingolani G, Alnemri ES. Cleavage of DFNA5 by caspase-3 during apoptosis mediates progression to secondary necrotic/pyroptotic cell death. Nat Commun [Internet]. Nature Publishing Group; 2017;8:1-14. Available from: http://dx.doi.org/10.1038/ncomms14128.

10. 10.1038 /onc. 2016.302

Bernardini JP, Lazarou M, Dewson G. Parkin and mitophagy in cancer. Oncogene [Internet]. Nature Publishing Group; 2017;36:1315-27. Available from: http://dx.doi.org/10.1038/onc.2016.302.

11. Drake LE, Springer MZ, Poole LP, Kim CJ, Macleod KF. Expanding perspectives on the significance of mitophagy in cancer. Semin Cancer Biol [Internet]. 2017;47:110-24. Available from: https://linkinghub.elsevier.com/retrieve/pii/S1044579X17301025.

12. $10.1016 /$ j.freeradbiomed.2017.01.004

Galadari S, Rahman A, Pallichankandy S, Thayyullathil F. Reactive oxygen species and cancer paradox: To promote or to suppress? Free Radic Biol Med [Internet]. Elsevier B.V.; 2017;104:144-64. Available from: https://doi.org/10.1016/j.freeradbiomed.2017.01.004.

13. 10.1016/j.semcdb.2017.05.023

Moloney JN, Cotter TG. ROS signalling in the biology of cancer. Semin Cell Dev Biol [Internet]. Elsevier Ltd; 2018;80:50-64. Available from: http://dx.doi.org/10.1016/j.semcdb.2017.05.023.

14. Wiśniewski JR, Zougman A, Nagaraj N, Mann M. Universal sample preparation method for proteome analysis. Nat Methods. 2009;6:359-62.

15. Cox J, Hein MY, Luber CA, Paron I, Nagaraj N, Mann M. Accurate proteome-wide label-free quantification by delayed normalization and maximal peptide ratio extraction, termed MaxLFQ. Mol Cell Proteomics. 2014;13:2513-26.

16. Sosna J, Voigt S, Mathieu S, Lange A, Thon L, Davarnia P, et al. TNF-induced necroptosis and PARP1-mediated necrosis represent distinct routes to programmed necrotic cell death. Cell Mol Life Sci. 2014;71:331-48.

17. Zhang K, Chen D, Ma K, Wu X, Hao H, Jiang S. NAD(P)H:Quinone Oxidoreductase 1 (NQO1) as a Therapeutic and Diagnostic Target in Cancer. J Med Chem. 2018;61:6983-7003.

18. 10.1016/j.jep.2016.08.045

Jiang G, Liu J, Ren B, Tang Y, Owusu L, Li M, et al. Anti-tumor effects of osthole on ovarian cancer cells in vitro. J Ethnopharmacol [Internet]. Elsevier; 2016;193:368-76. Available from: http://dx.doi.org/10.1016/j.jep.2016.08.045.

19. Zhu X, Song X, Xie K, Zhang X, He W, Liu F. Osthole induces apoptosis and suppresses proliferation via the PI3K/Akt pathway in intrahepatic cholangiocarcinoma. Int J Mol Med. 2017;40:1143-51.

20. Xu X, Zhang Y, Qu D, Jiang T, Li S. Osthole induces G2/M arrest and apoptosis in lung cancer A549 cells by modulating PI3K/Akt pathway. J Exp Clin Cancer Res. 2011;30:1-7.

21. 10.1016/j.ejphar.2020.172990 
Liang J, Zhou J, Xu Y, Huang X, Wang X, Huang W, et al. Osthole inhibits ovarian carcinoma cells through LC3-mediated autophagy and GSDME-dependent pyroptosis except for apoptosis. Eur J Pharmacol [Internet]. Elsevier B.V.; 2020;874:172990. Available from:

https://doi.org/10.1016/j.ejphar.2020.172990.

22. $10.1038 /$ nature22393

Wang Y, Gao W, Shi X, Ding J, Liu W, He H, et al. Chemotherapy drugs induce pyroptosis through caspase-3 cleavage of a gasdermin. Nature [Internet]. Nature Publishing Group; 2017;547:99-103. Available from: http://dx.doi.org/10.1038/nature22393.

23. Mai FY, He P, Ye JZ, Xu LH, Ouyang DY, Li CG, et al. Caspase-3-mediated GSDME activation contributes to cisplatin- and doxorubicin-induced secondary necrosis in mouse macrophages. Cell Prolif. 2019;52:1-11.

24. Oh ET, Park HJ. Implications of NQO1 in cancer therapy. BMB Rep. 2015;48:609-17.

25. 10.1016/j.freeradbiomed.2020.03.026

Yu J, Zhong B, Jin L, Hou Y, Ai N, Ge W, et al. 2-Methoxy-6-acetyl-7-methyljuglone (MAM) induced programmed necrosis in glioblastoma by targeting NAD $(\mathrm{P}) \mathrm{H}$ : Quinone oxidoreductase 1 (NQ01). Free Radic Biol Med [Internet]. Elsevier B.V.; 2020;152:336-47. Available from: https://doi.org/10.1016/j.freeradbiomed.2020.03.026.

26. Park W, Park S, Song G, Lim W. Inhibitory effects of osthole on human breast cancer cell progression via induction of cell cycle arrest, mitochondrial dysfunction, and er stress. Nutrients. 2019;11.

27. Ueda S, Masutani H, Nakamura H, Tanaka T, Ueno M, Yodoi J. Redox control of cell death. Antioxidants Redox Signal. 2002;4:405-14.

28. Soldani C, Lazzè MC, Bottone MG, Tognon G, Biggiogera M, Pellicciari CE, et al. Poly(ADP-ribose) polymerase cleavage during apoptosis: When and where? Exp Cell Res. 2001;269:193-201.

29. 10.1038/s41419-019-1441-4

Yu J, Li S, Qi J, Chen Z, Wu Y, Guo J, et al. Cleavage of GSDME by caspase-3 determines lobaplatininduced pyroptosis in colon cancer cells. Cell Death Dis [Internet]. Springer US; 2019;10. Available from: http://dx.doi.org/10.1038/s41419-019-1441-4.

30. Kim I, Rodriguez-Enriquez S, Lemasters JJ. Selective degradation of mitochondria by mitophagy. Arch Biochem Biophys. 2007;462:245-53.

31. 10.1038/s41419-019-1470-z

Yao N, Wang C, Hu N, Li Y, Liu M, Lei Y, et al. Inhibition of PINK1/Parkin-dependent mitophagy sensitizes multidrug-resistant cancer cells to B5G1, a new betulinic acid analog. Cell Death Dis [Internet]. Springer US; 2019;10. Available from: http://dx.doi.org/10.1038/s41419-019-1470-z.

32. Li L, Tan J, Miao Y, Lei P, Zhang Q. ROS and Autophagy: Interactions and Molecular Regulatory Mechanisms. Cell Mol Neurobiol. 2015;35:615-21.

33. 10.1016/j.redox.2019.101254

Lin Q, Li S, Jiang N, Shao X, Zhang M, Jin H, et al. PINK1-parkin pathway of mitophagy protects against contrast-induced acute kidney injury via decreasing mitochondrial ROS and NLRP3 
inflammasome activation. Redox Biol [Internet]. Elsevier B.V.; 2019;26:101254. Available from: https://doi.org/10.1016/j.redox.2019.101254.

\section{Figures}

A

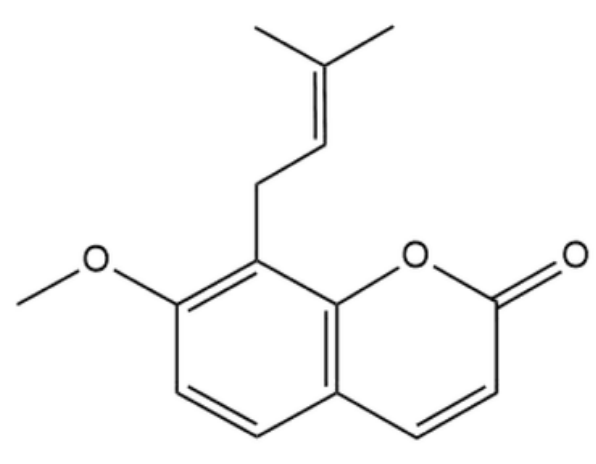

Osthole

C

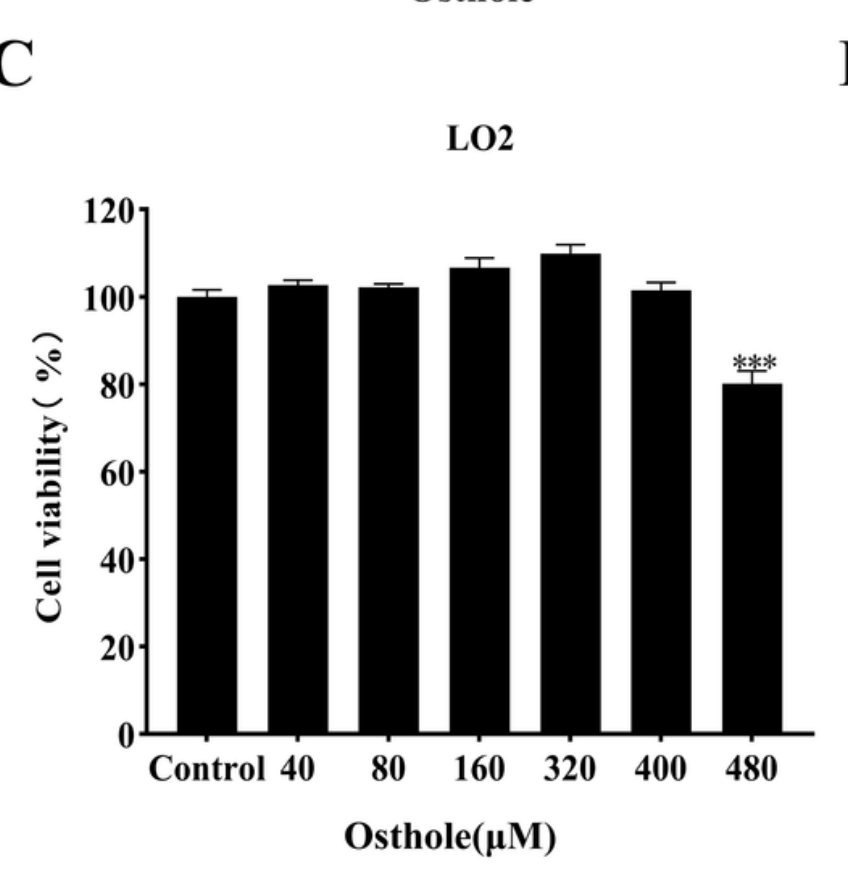

B

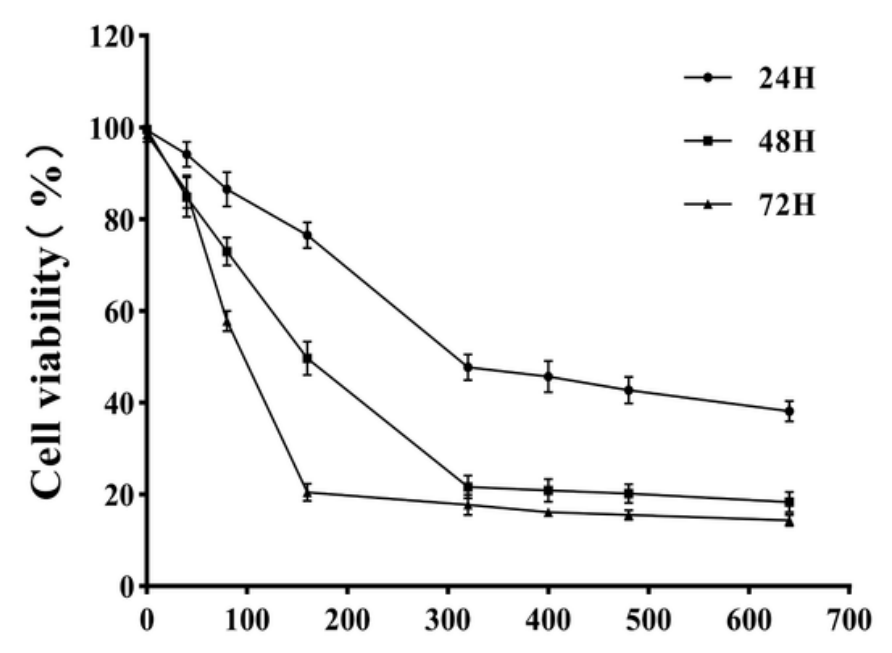

Osthole $(\mu \mathrm{M})$
D

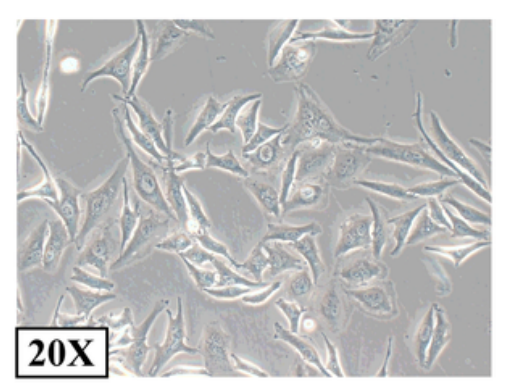

Control

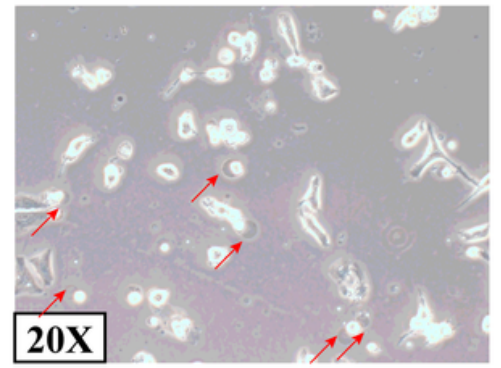

160

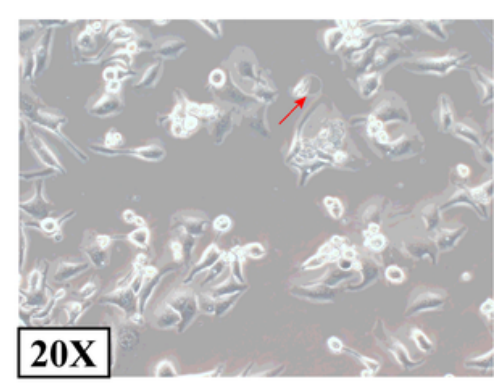

80

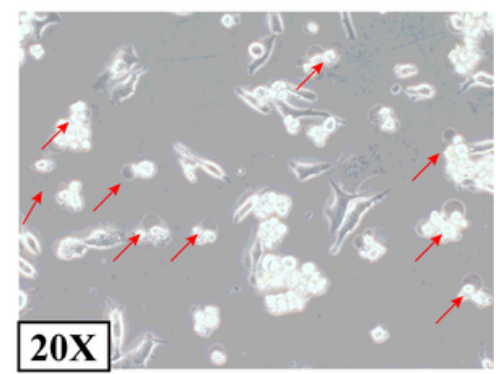

320
Osthole $(\mu \mathrm{M})$

Figure 1

Osthole inhibited the cell viability and induced cell death in HeLa cells. (A) The chemical structural formula of osthole. (B) MTT assay showed that the cell vitality was decreased by degrees after treated with osthole at advisable time for advisable time. (C) The cytotoxic effect on normal liver LO2 cells was detected by MTT assay after treated with osthole for $18 \mathrm{~h}$. (D) Representative bright-field images were 
shot after different concentrations osthole treated for $18 \mathrm{~h}$ in HeLa cells (20X). Red arrowheads indicated the large bubbles emerging from the plasma membrane. Data are represented as means $\pm S D, n=3$, $\star \star \star P$ $<0.001$ compared with the control group.

A

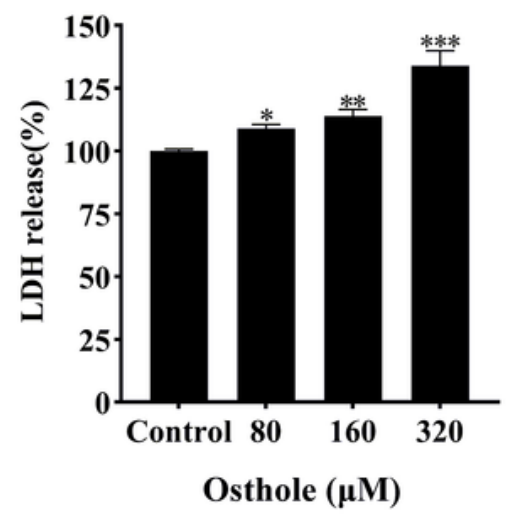

C

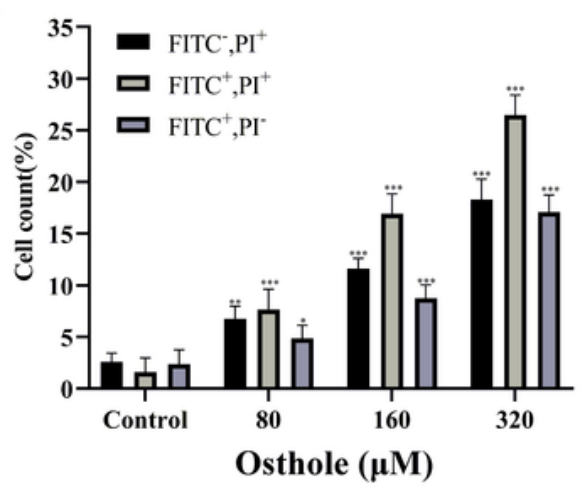

D

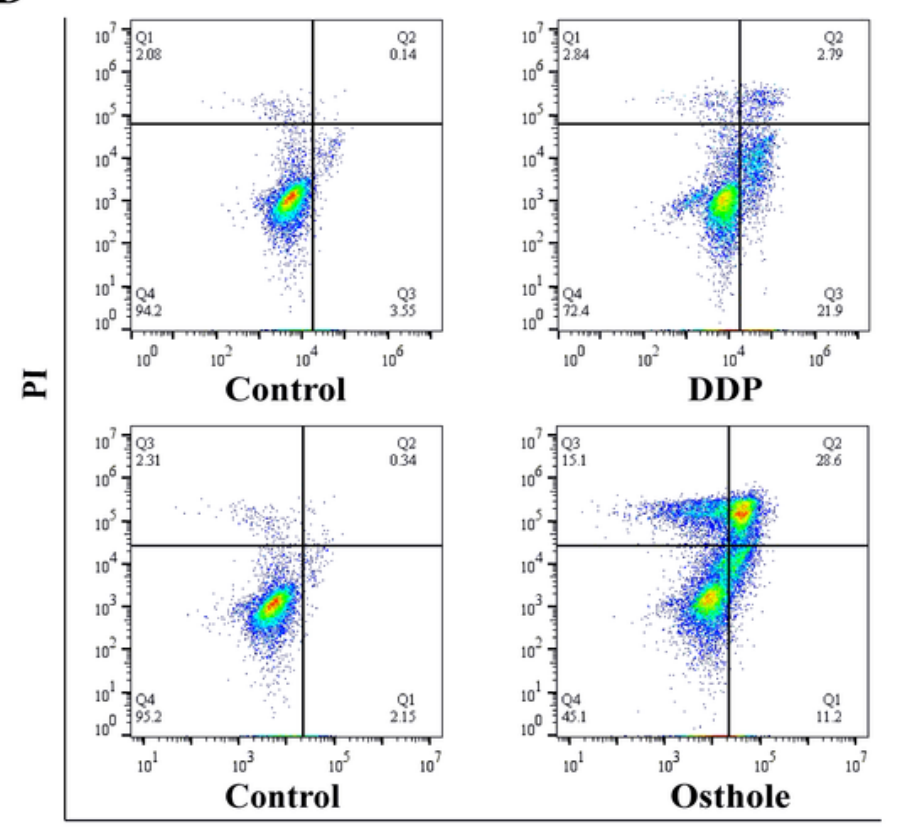

FITC
B
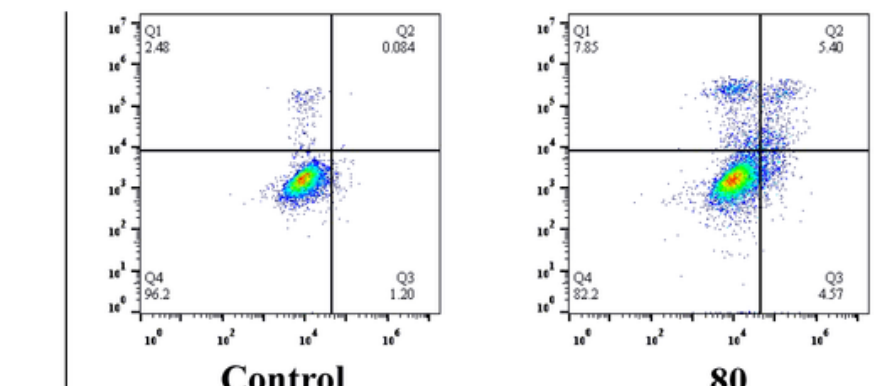

80

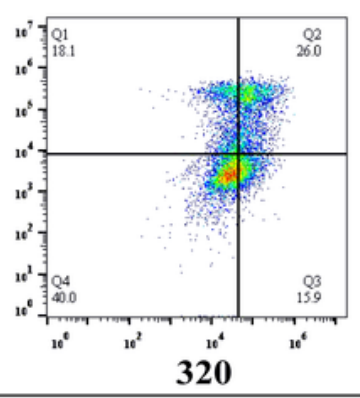

ITC

Osthole $(\mu \mathrm{M})$

E

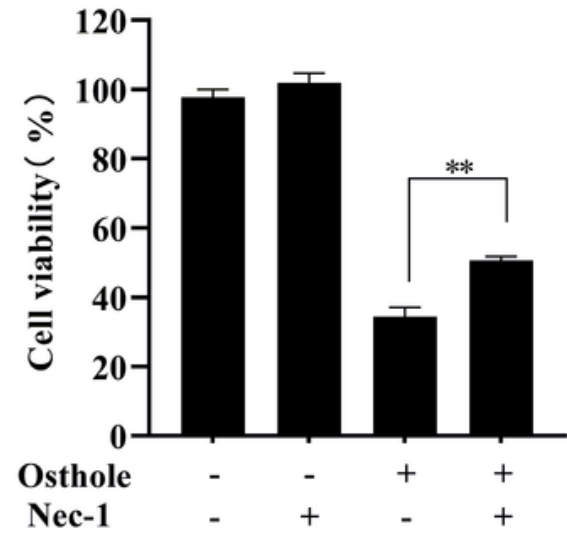

$\mathbf{F}$

RIP3

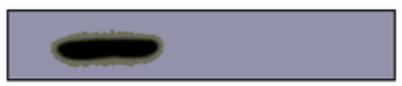

$\beta$-actin

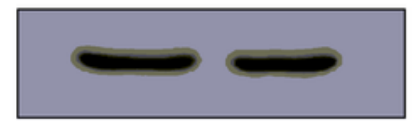

U87

Hela

Figure 2

Osthole induced apoptosis and necrosis in RIP3-deficient HeLa cells. (A) The release of LDH was detected after treated with indicated concentration for $18 \mathrm{~h}$. (B) After treatment with indicated concentration the 
cell death was detected using Annexin囚-FITC/ PI double staining by flow cytometry in HeLa cells. (C) Ratio of cells stained by FITC and/or PI. (D) Comparison of osthole-induced cell death and DDP-induced cell death. (E) Pretreatment with $100 \mu \mathrm{M} \mathrm{Nec}-1$ for $1 \mathrm{~h}$ before treatment with $320 \mu \mathrm{M}$ osthole for $18 \mathrm{~h}$, the cell vitality was detected by MTT assay. (F) The expression of RIP3 was tested by western blotting assay in HeLa and U87 cells. Data are represented as means $\pm S D, n=3, * P<0.05,{ }^{\star *} P<0.01, \star \star \star P<0.001$ compared with the control group.

A
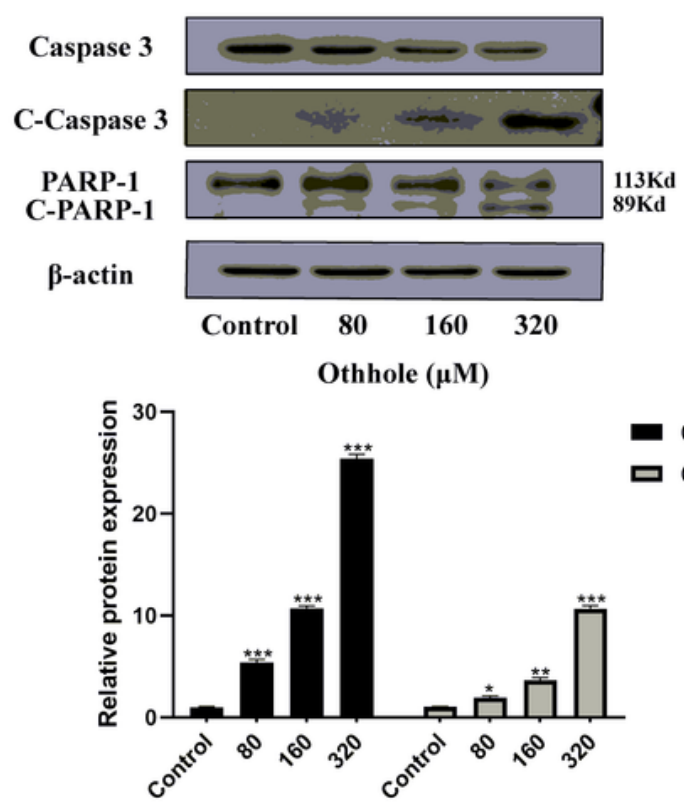

Othhole $(\mu \mathrm{M})$

C

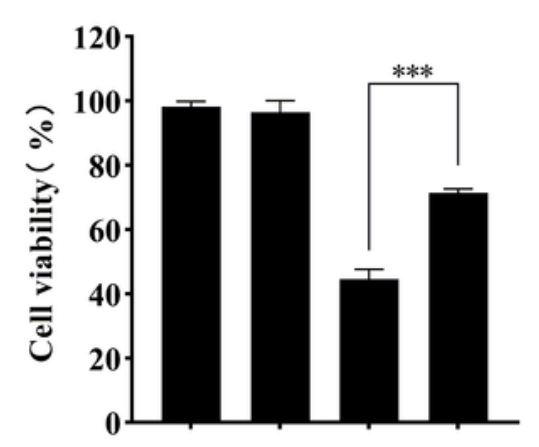

$\mathbf{E}$
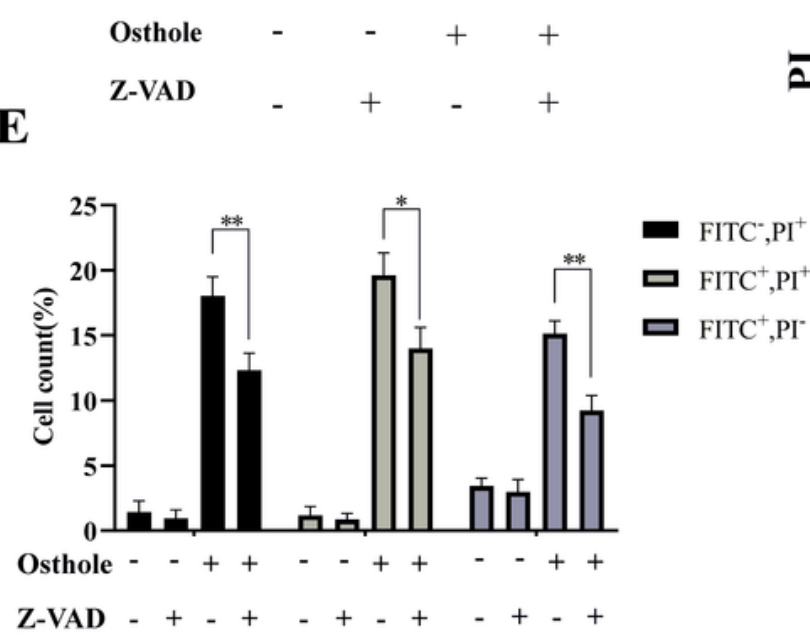

B

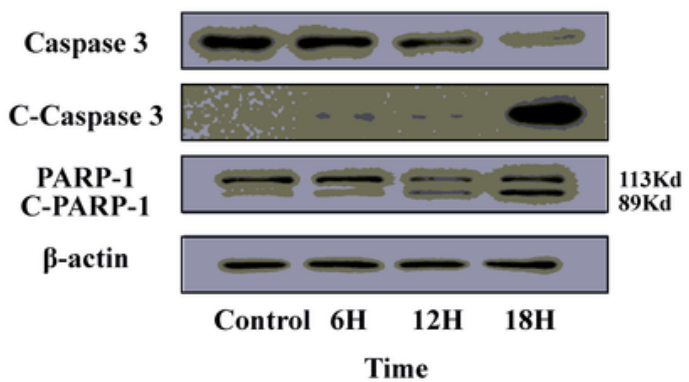

C-Caspase 3

C-PARP-1

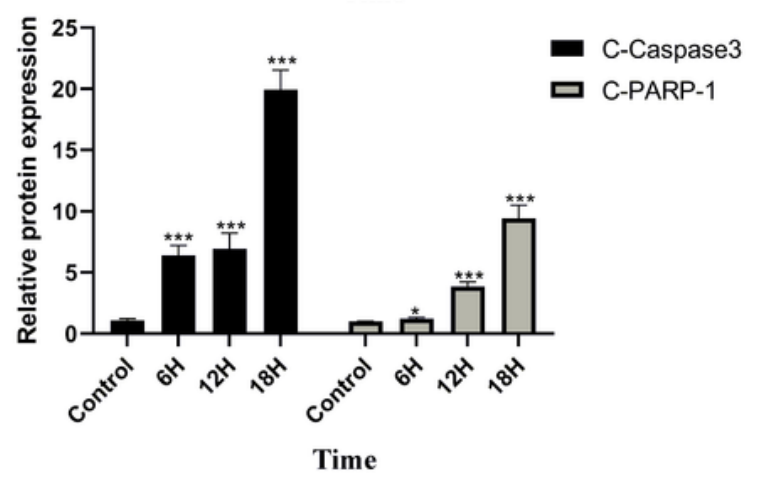

D

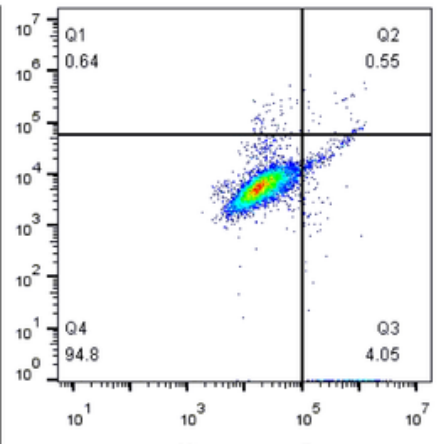

Control
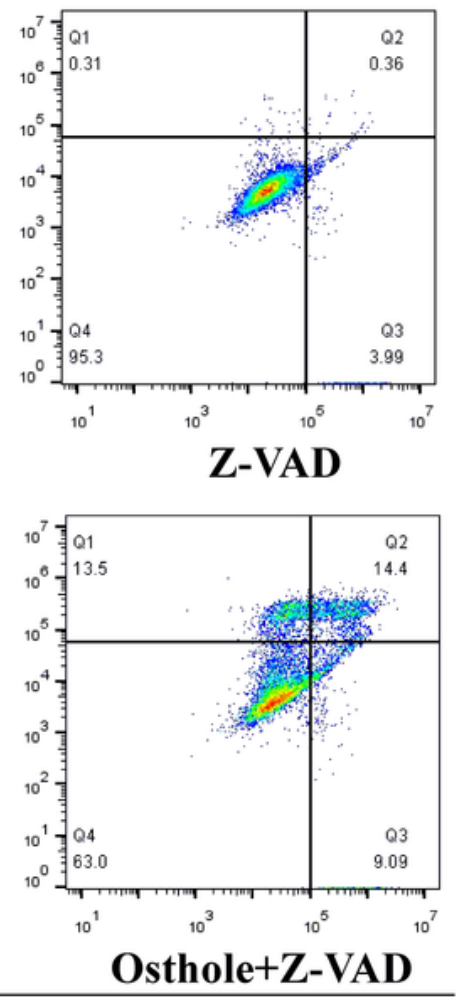

FITC

Figure 3 
Osthole induced caspase-mediated apoptosis and necrosis in HeLa cells. (A, B) After treatment with indicated concentration of osthole for $18 \mathrm{~h}$ or $320 \mu \mathrm{M}$ osthole for indicated time, the levels of apoptosisrelated protein were determined by western blotting. The intensity of bands was quantified by image $\mathrm{J}$, and $\beta$-actin was used as a control. (C-E) Pretreatment with 20 $\mathrm{M}$ Z-VAD-FMK for $1 \mathrm{~h}$ prior to treatment with $320 \mu \mathrm{M}$ osthole for $18 \mathrm{~h}$. (C) Cell vitality was detected by MTT assay. (D) The form of cell death was determined using Annexin $\triangle-F I T C / P I$ double staining by flow cytometry. (E) Ratio of cells stained by FITC and/or PI. All Data are represented as means $\pm \mathrm{SD}, \mathrm{n}=3$, ${ }^{\star \mathrm{P}}<0.05$, ${ }^{\star *} \mathrm{P}<0.01$, ${ }^{\star \star \star} \mathrm{P}<0.001$ compared with the control group. 
A

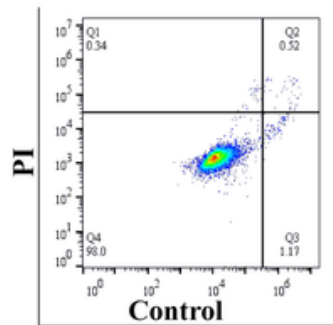

Time

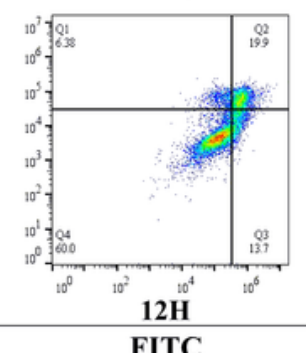

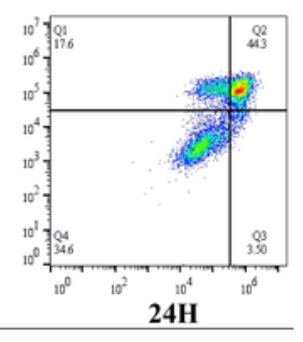

D

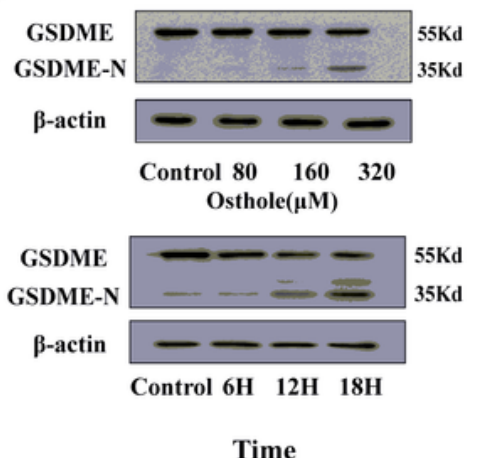

Time

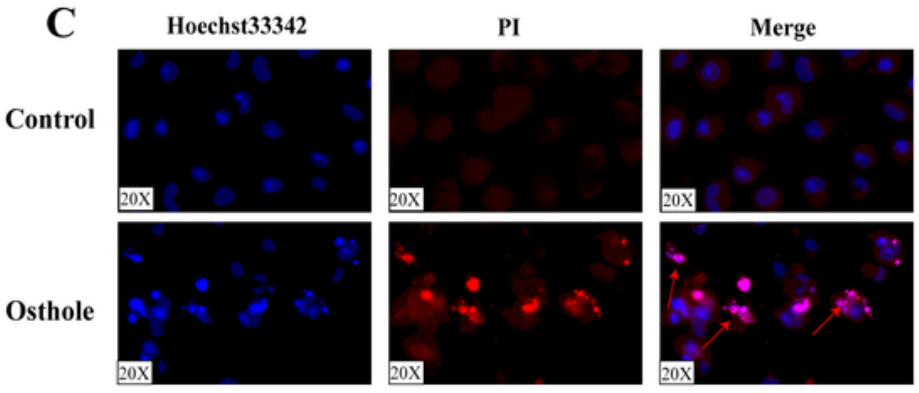

E

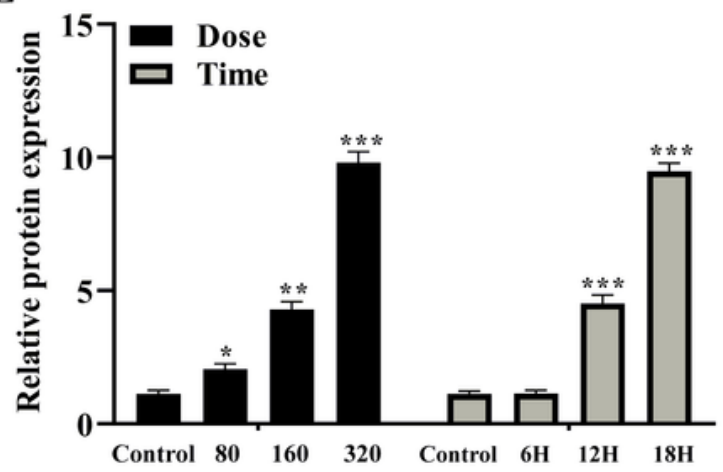

H

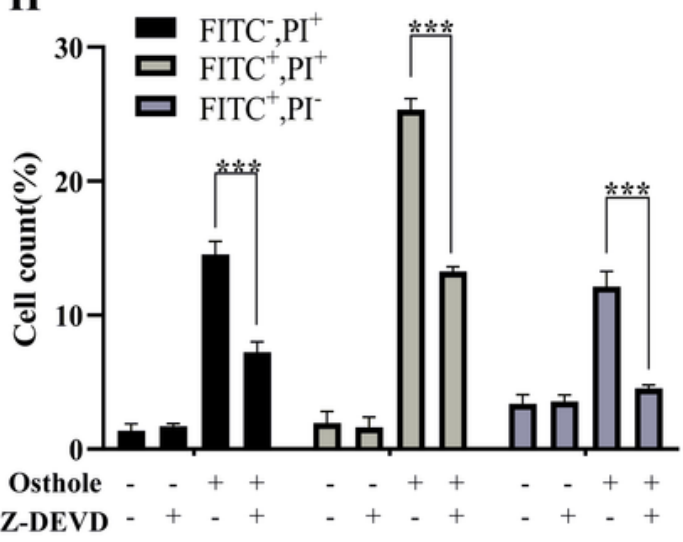

F

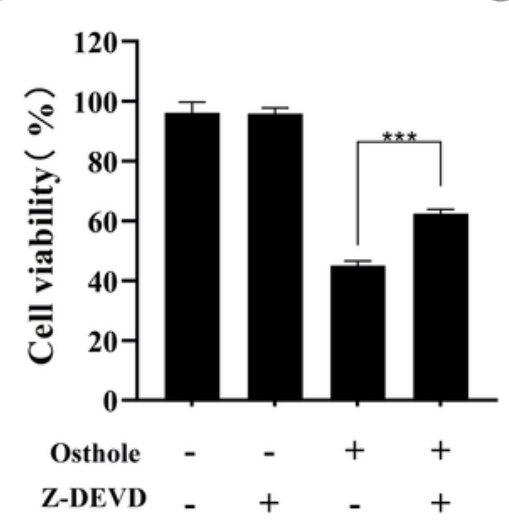

I

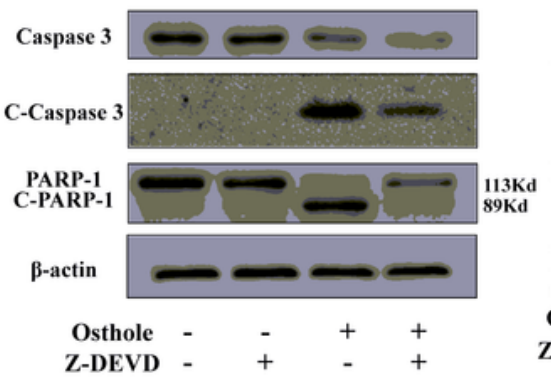

G

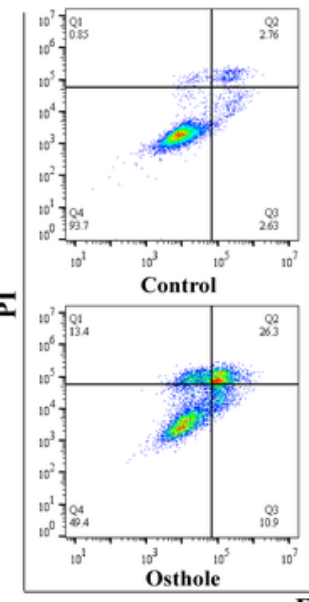

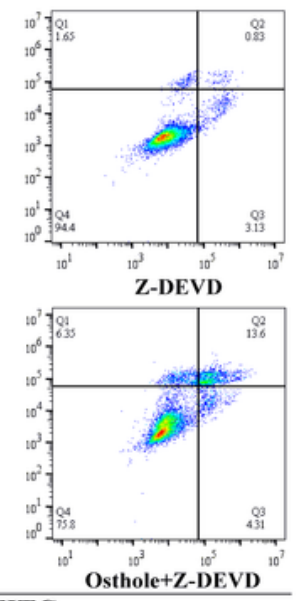

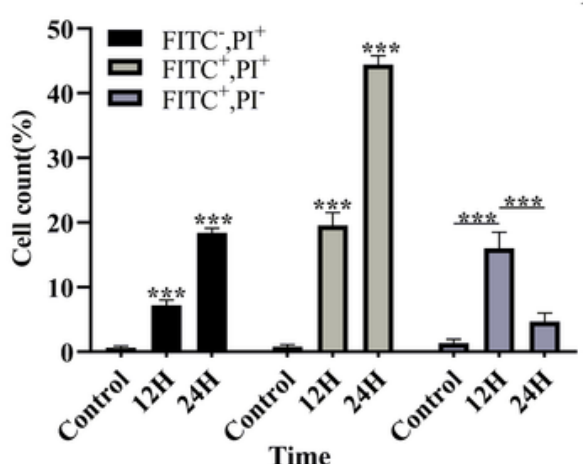

\section{FITC}
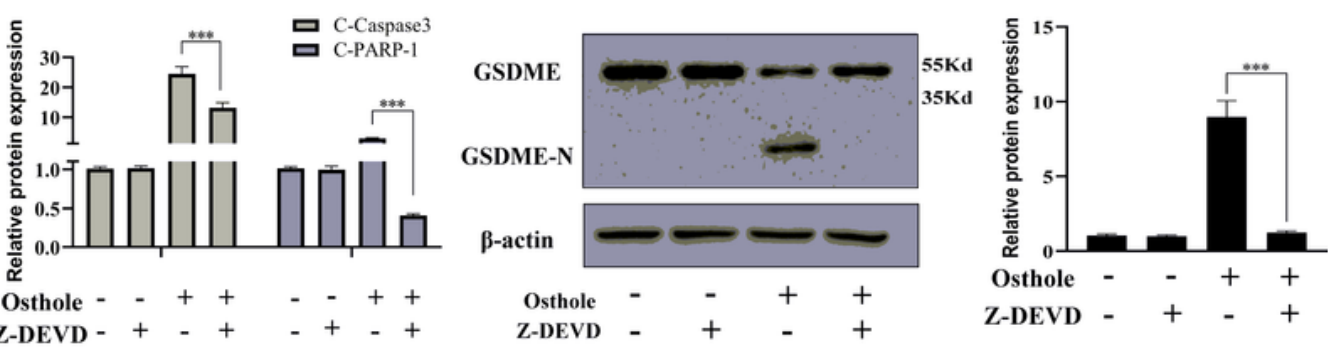

\section{Figure 4}

Osthole induced Caspase-3-mediated apoptosis and secondary necrosis in HeLa cells. (A) Annexin 》FITC/PI double staining analysis showed that the transformation of FITC and PI staining with the time increased after treated with $320 \mu \mathrm{M}$ osthole. (B) Ratio of cells stained by FITC and/or PI. (C) Representative images of Hoechst 33342/PI double staining showed the apoptosis and necrosis cells (20X). (D) Treatment with indicated concentration for $18 \mathrm{~h}$ or $320 \mu \mathrm{M}$ osthole for indicated time. The 
expression of GSDME and GSDME-N was analyzed by western blotting at protein levels. (E) The intensity of bands was quantified by image $\mathrm{J}$, and $\beta$-actin was used as a control. (F-J) Pretreatment with $50 \mu \mathrm{M} \mathrm{Z-}$ DEVD-FMK for $1 \mathrm{~h}$ prior to treatment with $320 \mu \mathrm{M}$ osthole (F) MTT assay detected the cell vitality. (G) Annexin X-FITC/PI double staining analyzed the cell death. (H) Ratio of cells stained by FITC and/or PI. (I) The expression of apoptosis-related proteins was detected by western blotting. The intensity of bands was quantified by image $\mathrm{J}$, and $\beta$-actin was used as a control. $(\mathrm{J})$ The expression of GSDME and GSDME$\mathrm{N}$ was detected by western blotting at protein levels. The intensity of bands was quantified by image $J$, and $\beta$-actin was used as a control. All Data are represented as means $\pm S D, n=3$, $* \star * P<0.001$ compared with the control group. 
A

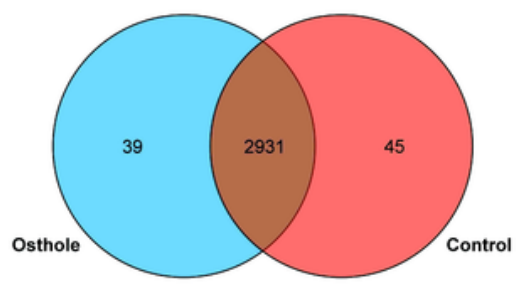

B

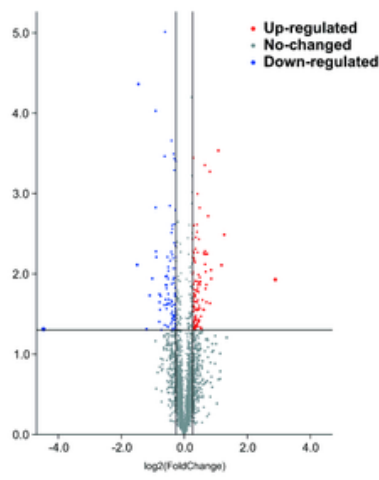

D

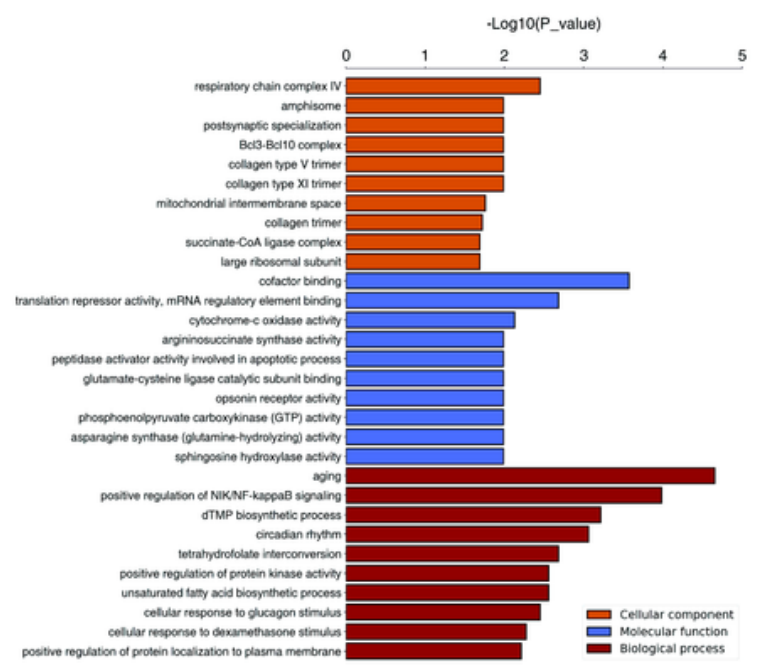

C

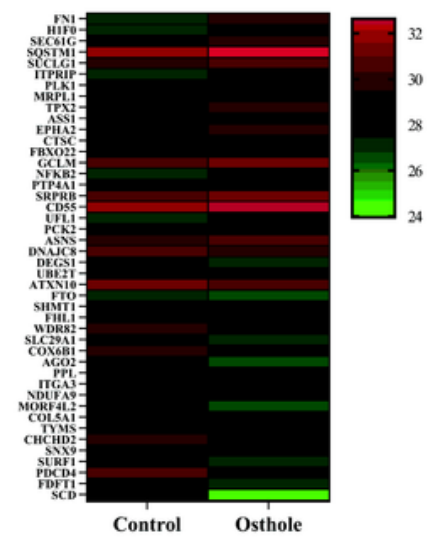

E

Metabolic pathways

Sesquiterpenoid and triterpenoid biosynthesis -

Biosynthesis of secondary metabolites

Alanine, aspartate and glutamate metabolism

Biosynthesis of amino acids

Biosynthesis of antibiotics

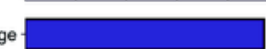

One carbon pool by folate

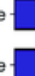

ay
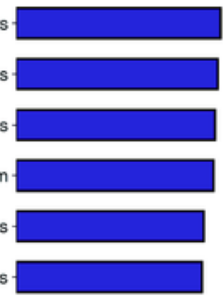

F

G
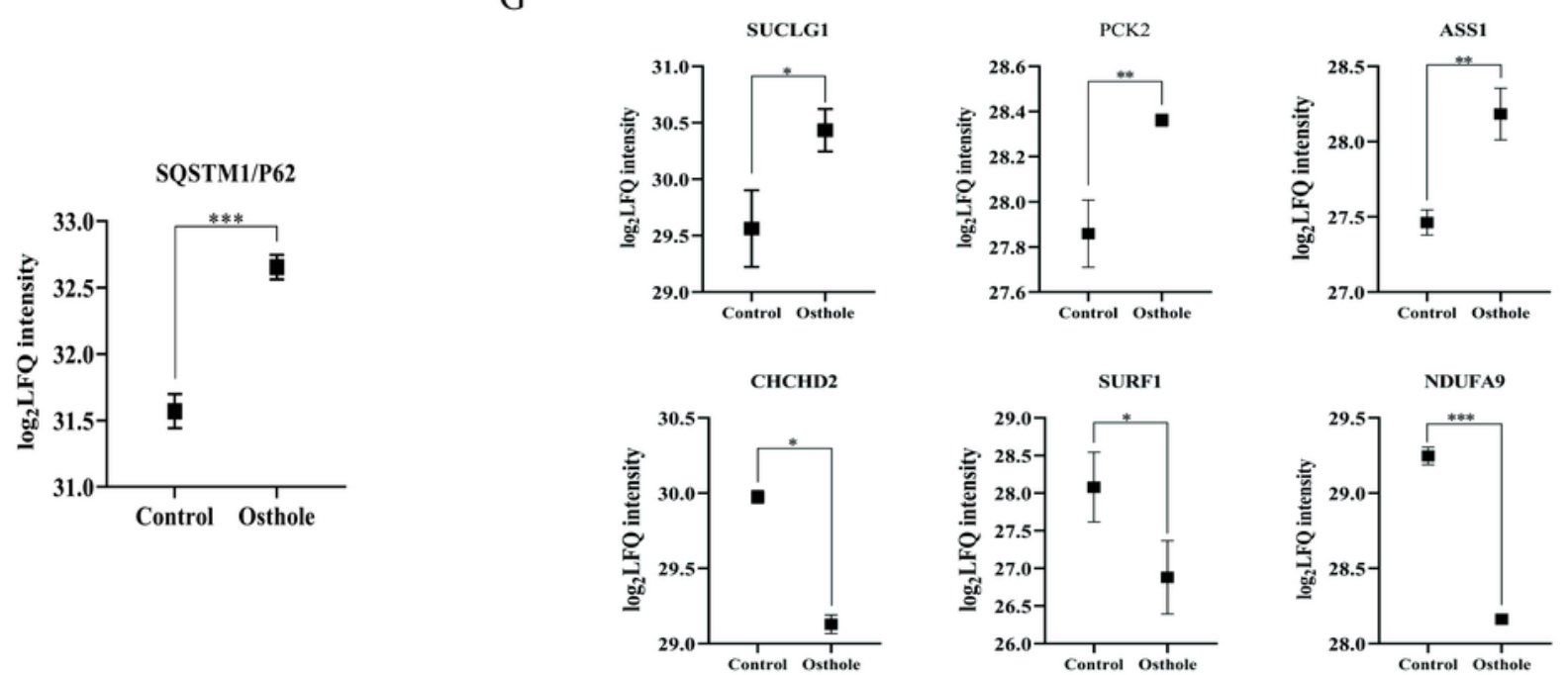

\section{Figure 5}

Label-Free Quantitative Proteomics analysis. (A) Venn diagram showed the coincident proteins and special protein between treated and untreated group. (B) Volcano plot showed the difference in expression between treated and untreated cells. Red dots represented the upregulated proteins and blue dots represented downregulated protein while grey dots represented unchanged proteins $(\log 2$ LFQ $\unrhd 1.2, P$ 『0.05). (C) Heat map of differentially expressed proteins (log2 LFQ $\otimes 1.2, P \otimes 0.05)$. (D) GO enrichment 
analysis of differentially expressed proteins. (F) KEGG pathway analysis of differentially expressed proteins. (G) Autophagy-related differentially expressed protein. $(\mathrm{H})$ Mitochondria-related differentially

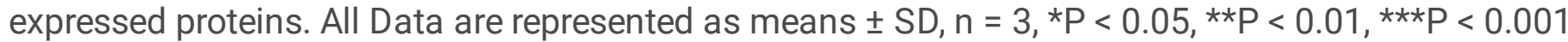
compared with the control group.

A

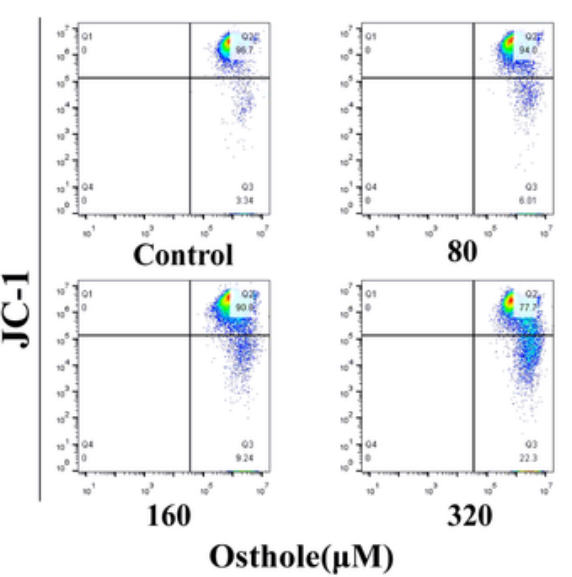

D

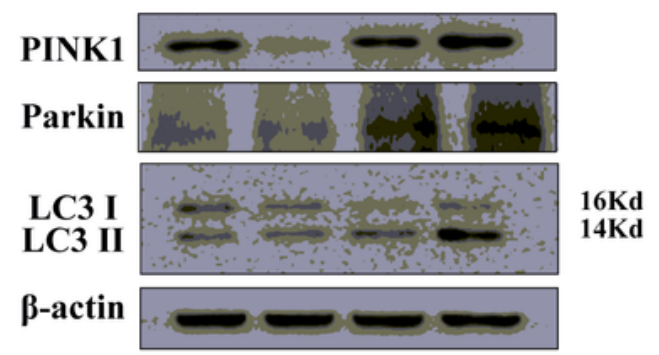

E
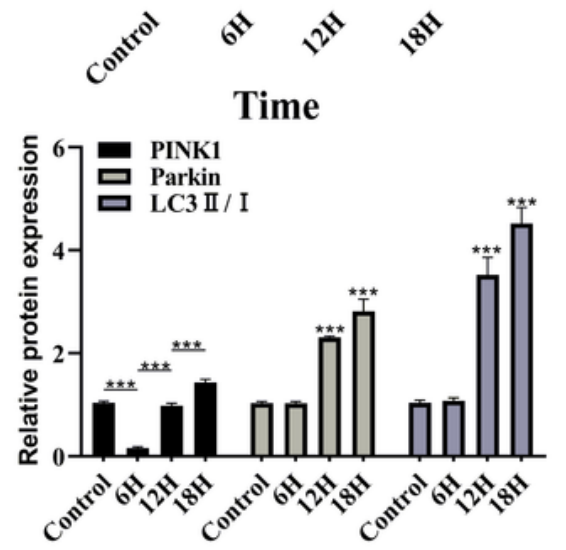

H

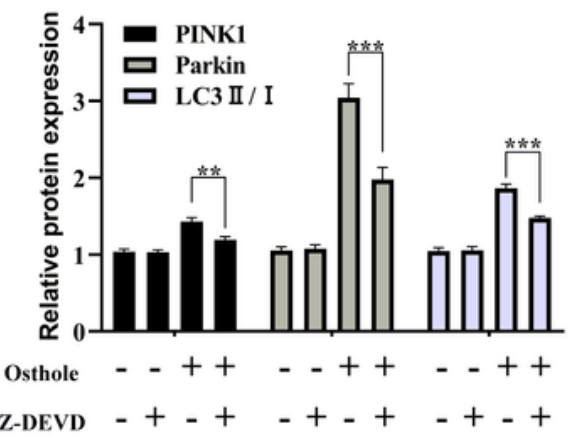

B

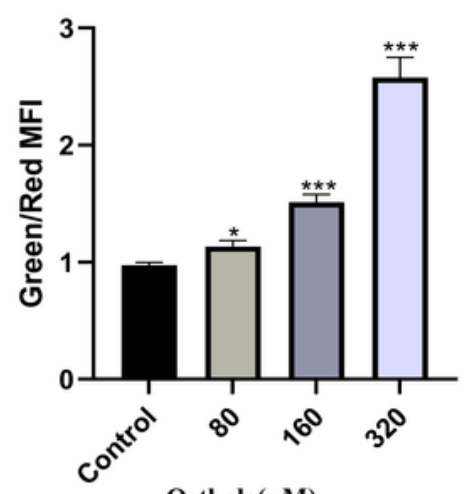

Osthole $(\mu \mathrm{M})$

$\mathbf{F}$

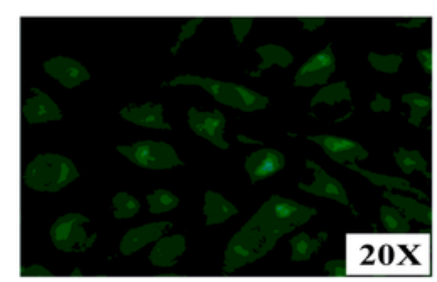

Control

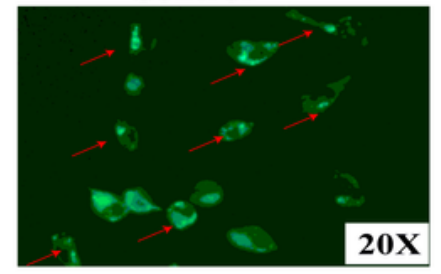

Osthole
C
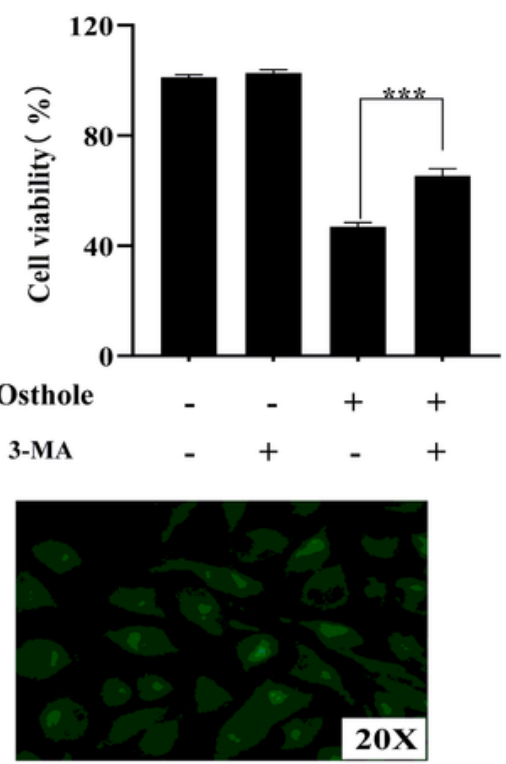

Z-DEVD

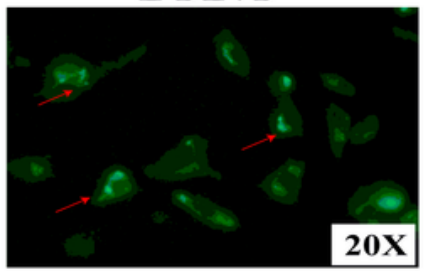

Osthole+Z-DEVD

G

PINK1

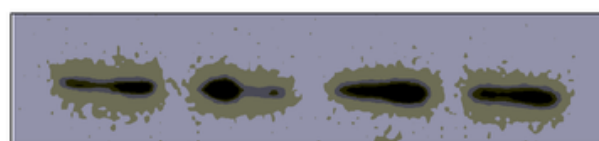

Parkin

LC3 I

LC3 II
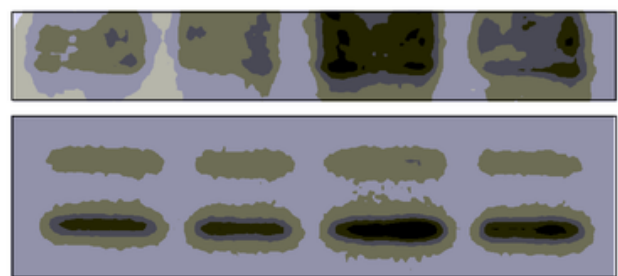

$16 \mathrm{Kd}$

$14 K d$

Figure 6 
Osthole induced caspase-3 mediated mitophagy in Hela cells. (A-B) Treatment with indicated concentration of osthole for $18 \mathrm{~h} \mathrm{(A)} \mathrm{The} \mathrm{JC-1} \mathrm{staining} \mathrm{was} \mathrm{analyzed} \mathrm{by} \mathrm{flow} \mathrm{cytometry.} \mathrm{(B)} \mathrm{The} \mathrm{ratio} \mathrm{of}$ mean fluorescence intensity of green fluorescence and red fluorescence quantified by FlowJo. (C) Pretreatment with $10 \mathrm{mM}$ 3-MA for $1 \mathrm{~h}$ prior to treatment with $320 \mu \mathrm{M}$ osthole, the cell vitality was detected by MTT assay. (D) Western blotting assay was used to detect the expression of mitophagyrelated proteins. (E) The intensity of bands was quantified by image $\mathrm{J}$, and $\beta$-actin was used as a control. (F-G) Pretreatment with Z-DEVD for $1 \mathrm{~h}$ prior to treatment $320 \mu \mathrm{M}$ osthole for $18 \mathrm{~h}$ (F) Representative images of MDC staining. Red arrows represent autophagic vacuoles. (G) The expression of mitophagymediated protein was detected by western blotting assay. $(\mathrm{H})$ The intensity of bands was quantified by image $J$, and $\beta$-actin was used as a control. All Data are represented as means $\pm S D, n=3, * P<0.05$, $* * P$

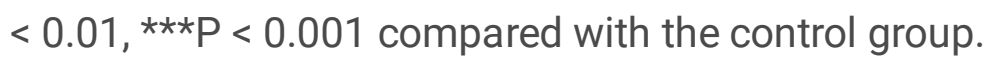


A

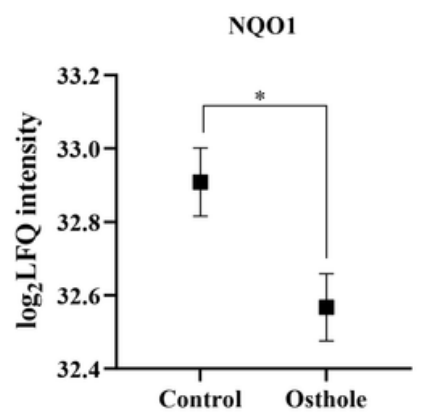

D
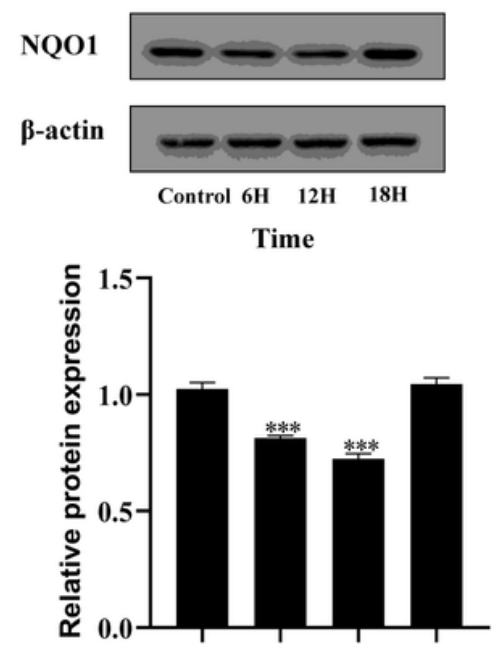

$c^{30}$

Time
B

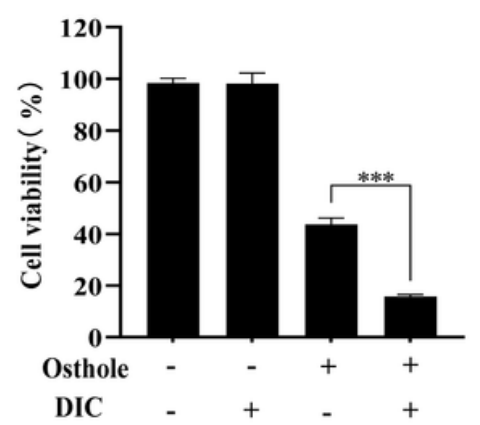

C

E
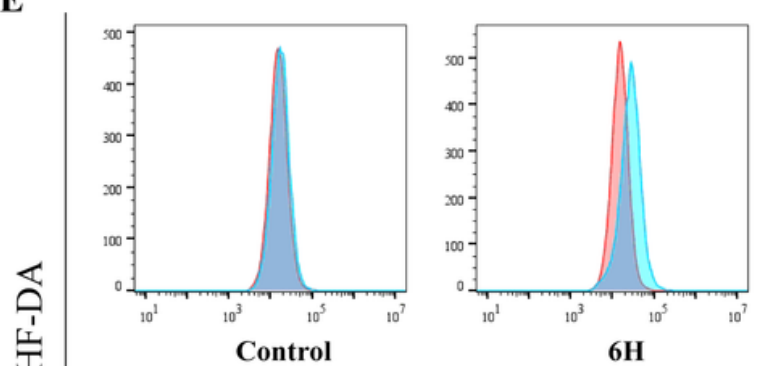

F

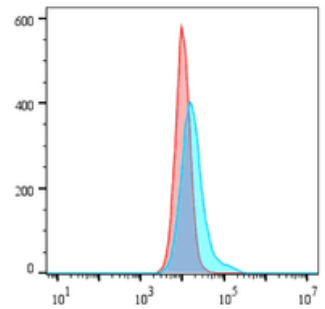

$12 \mathrm{H}$

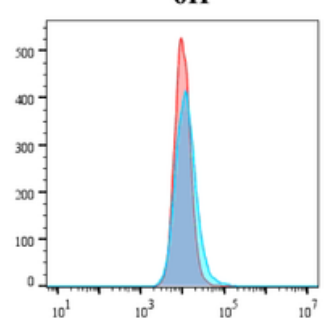

$18 \mathrm{H}$

Time

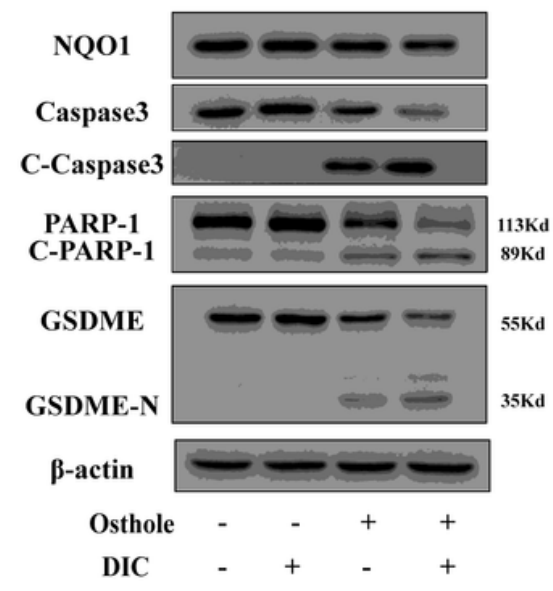

H

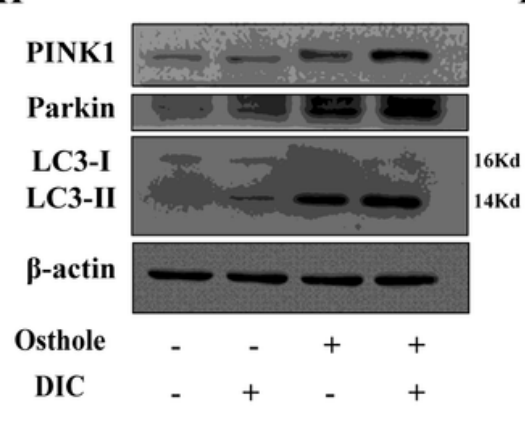

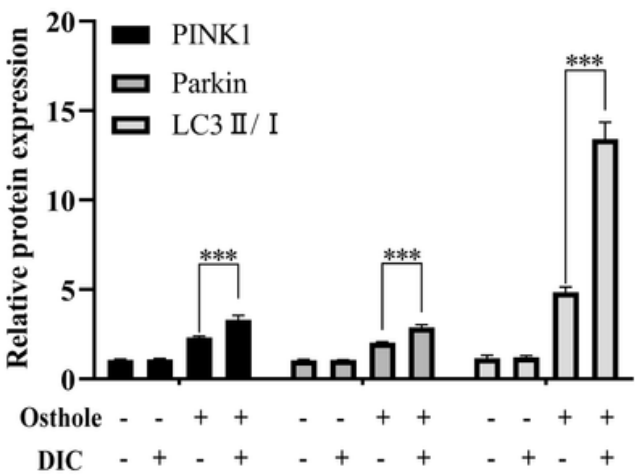

Figure 7

NQ01 contributed to the ROS overproduction and cell death in Hela cells. (A)The differences of NQ01 expression was detected by Label free proteomics analysis. (B-C and F-I) Incubation with $100 \mu \mathrm{M}$ DIC for $1 \mathrm{~h}$ ahead of treatment with $320 \mu \mathrm{M}$ osthole (B) Cell vitality was detected by MTT assay. (C) Treatment with osthole for $4 \mathrm{~h}$, DCFH-DA staining was detected by flow cytometry. (D-E) after treatment with $320 \mu \mathrm{M}$ osthole for indicated time. (D) The expression of NQ01 was detected by western blotting. (E) The generation of ROS was detected by DCHF-DA using flow cytometry. Red represents untrusted group and blue represents osthole-treated group $(F, G)$ Western blotting analyzed the expression of apoptosis-, 
necrosis- and mitophagy-related proteins. $(G, I)$ The intensity of bands was quantified by image $J$, and $\beta$ actin was used as a control. All Data are represented as means $\pm S D, n=3$, $P<<0.5,{ }^{\star \star} P<0.01$, $* \star \star P<$ 0.001 compared with the control group.
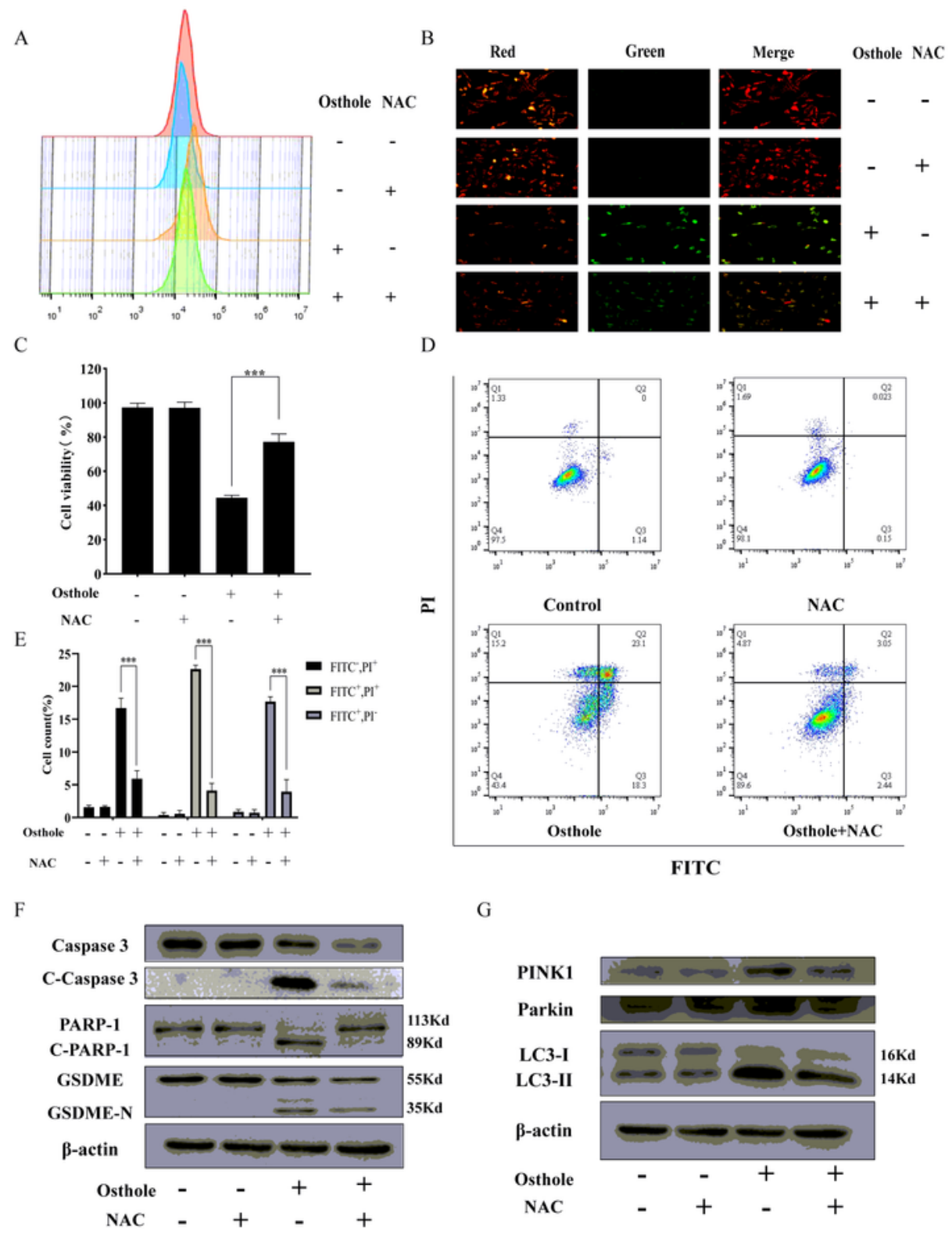

G
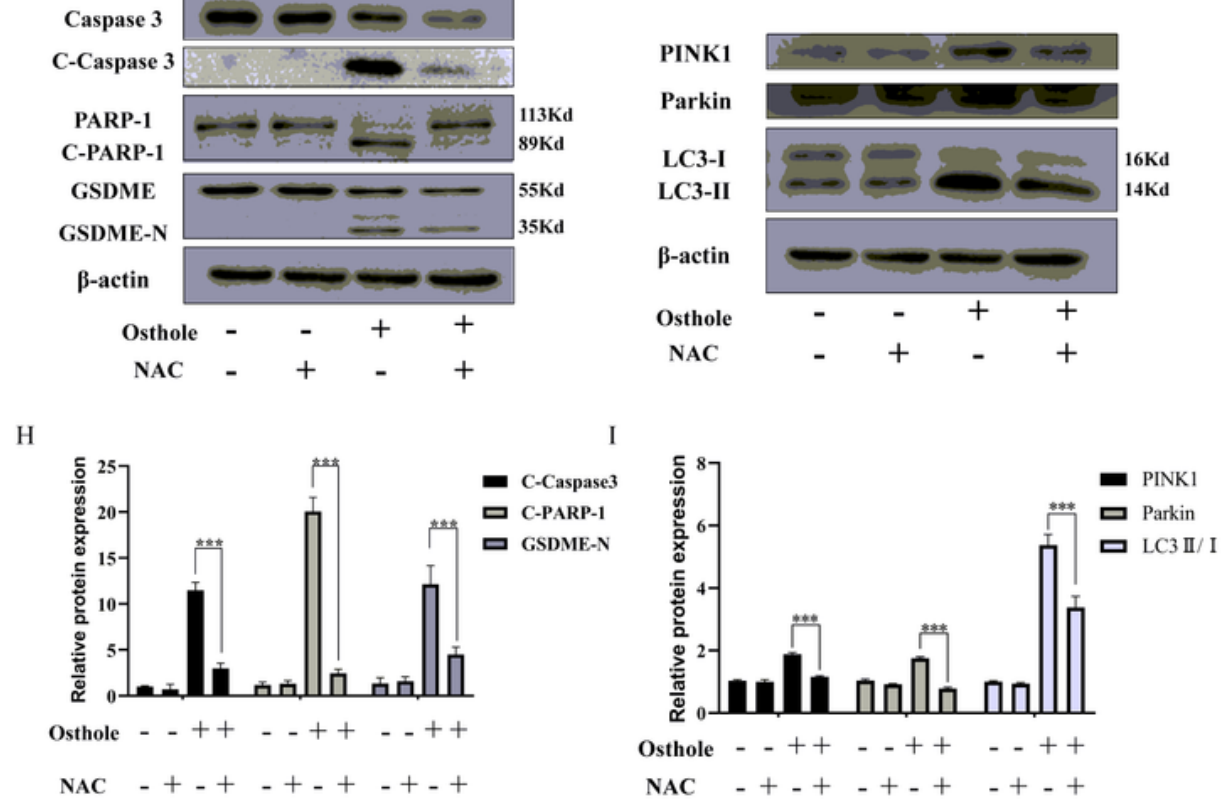

Figure 8

ROS contributed to the osthole-induced cell death in HeLa cells. (A-G) Pretreatment with $0.2 \mu \mathrm{M}$ NAC for $1 \mathrm{~h}$ prior to treatment with $320 \mu \mathrm{M}$ osthole (A) Treatment with osthole for 4h, DCHF-DA staining was 
detected by flow cytometry. (B) Representative images of JC-1 staining (20X). (C) Cell viability was

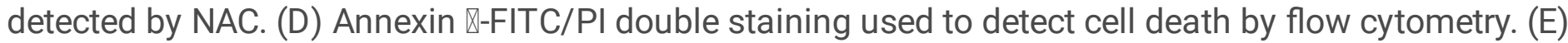
Ratio of cells stained by FITC and/or PI. (F and G) The expression of apoptosis-, necrosis- and mitophagy-related proteins was determined by western blotting. ( $\mathrm{H}$ and $\mathrm{I}$ ) The intensity of bands was quantified by image $J$, and $\beta$-actin was used as a control. All Data are represented as means $\pm S D, n=3$, $\star \star * P<0.001$ compared with the control group.

A

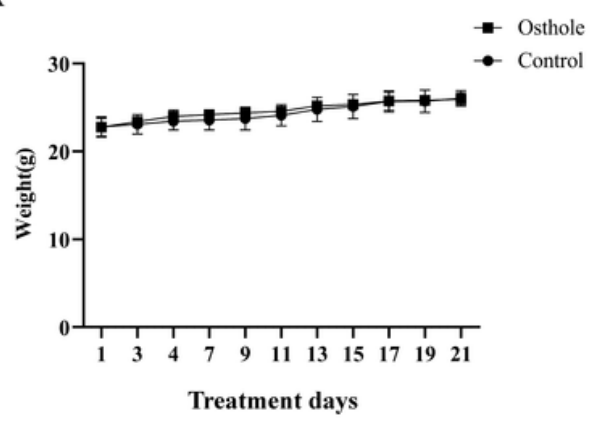

B

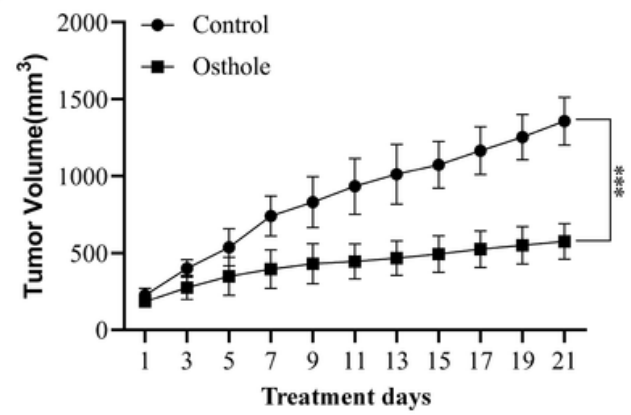

C

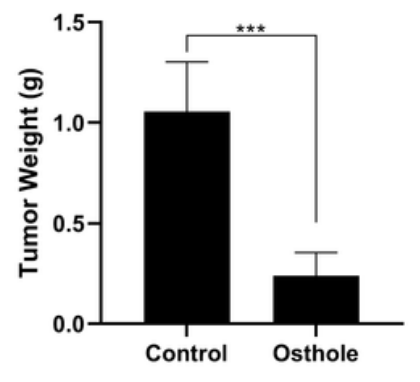

D

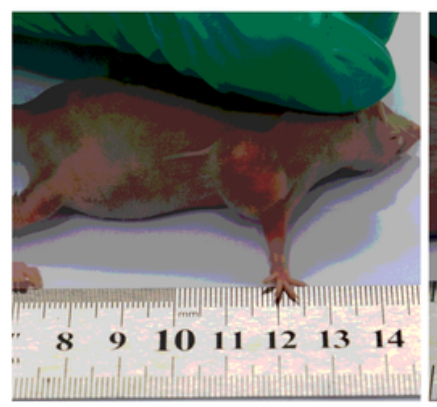

Control

$\mathbf{F}$

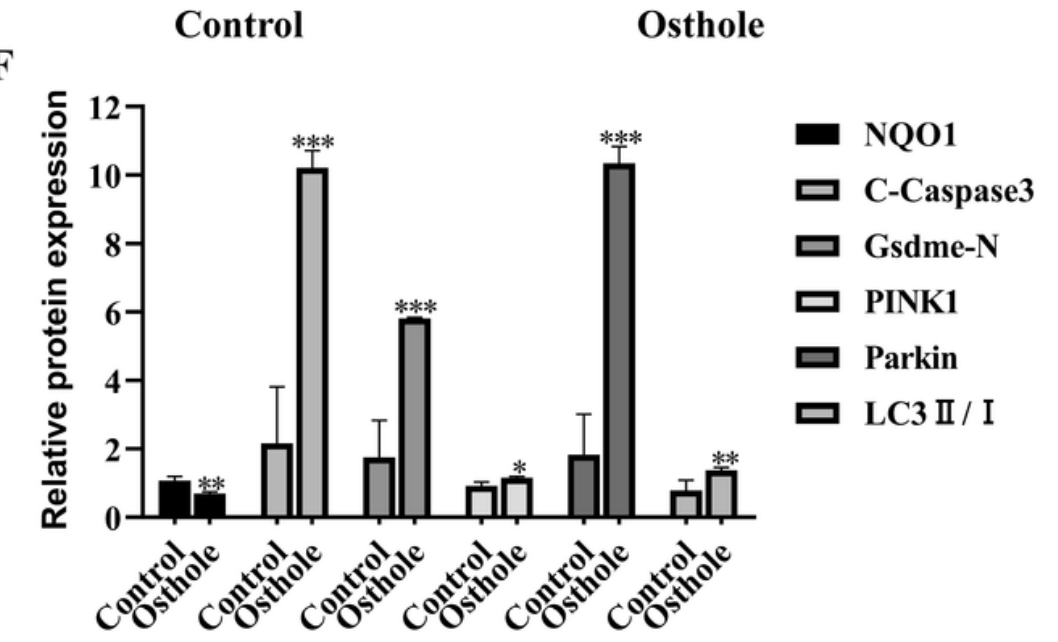

$\mathbf{E}$

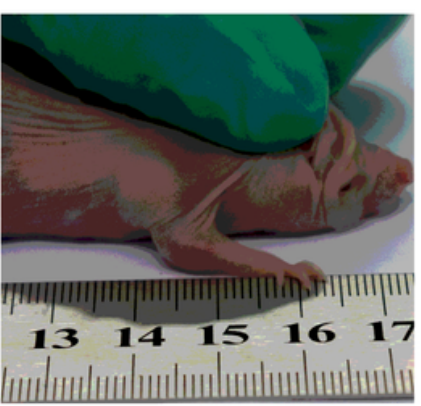

Osthole

\section{Figure 9}

Osthole suppressed HeLa xenograft tumor growth in vivo. (A) The body weight of nude mice treated with or without osthole for 21 days. (B) Tumor volume was recorded every 2 days. (C) The average weight of tumor in control and osthole treated group. (D) Representative images of unstripped tumor after treated for 21 days. (E) The expression of secondary necrosis- and mitophagy-related proteins was determined by western blotting. (F) The intensity of bands was quantified by image $J$, and $\beta$-actin was used as a 
control. All Data are represented as means $\pm S D, * P<0.001, * \star P<0.001, * \star * P<0.001$ compared with the control group.

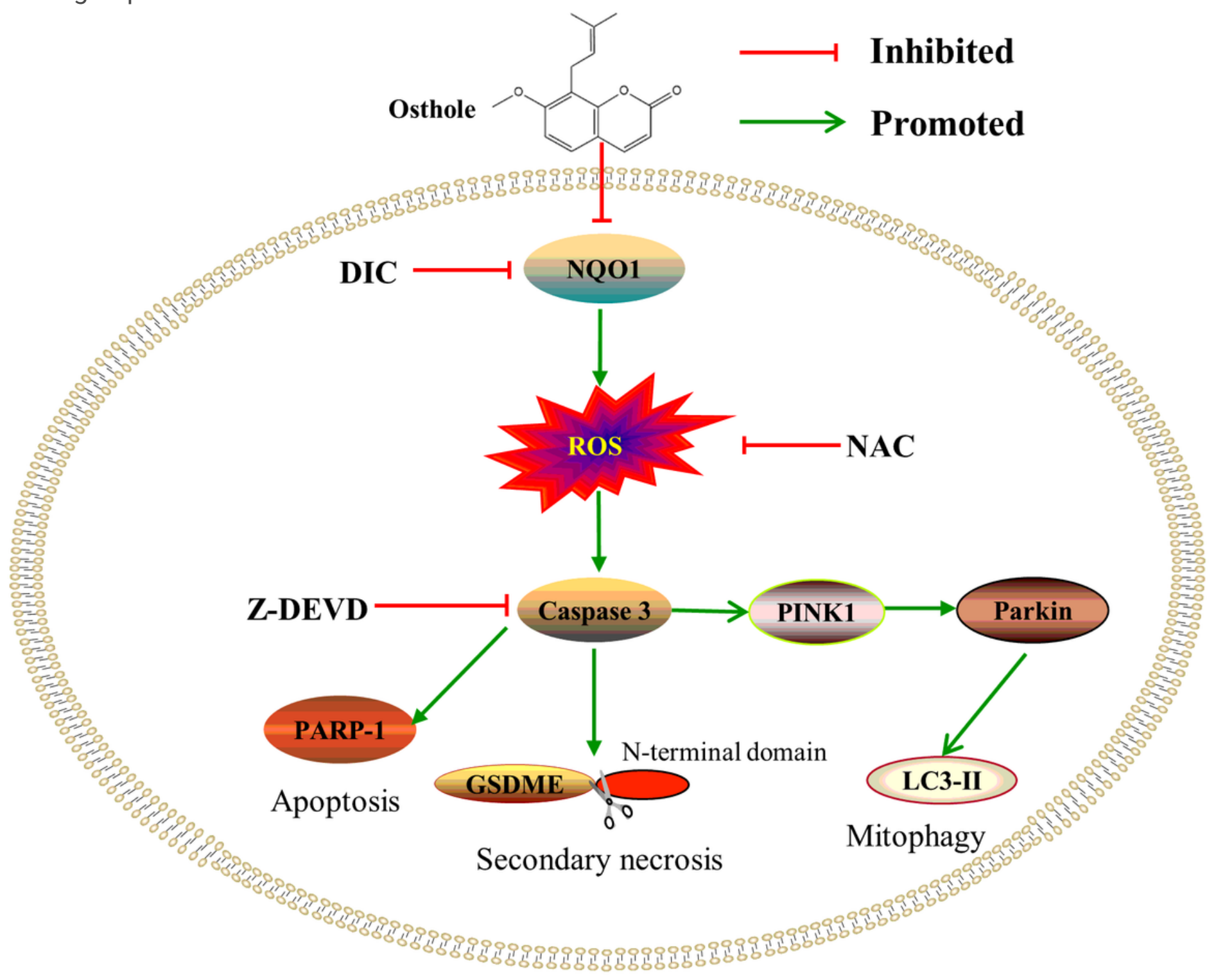

Figure 10

Schematic representation of ROS mediated osthole-induced apoptosis, secondary necrosis and mitophagy in HeLa cells.

\section{Supplementary Files}

This is a list of supplementary files associated with this preprint. Click to download.

- figs1.pdf

- TableS2Differentiallyexpressedproteins.xlsx

- TableS1IdentifiedProteins.xlsx 